\title{
Unified Description of the Specific Heat of Ionic Bulk Materials Containing Nanoparticles
}

\author{
E. Leonardi, ${ }^{\dagger}$ A. Floris, ${ }^{*}+$ S. Bose, ${ }^{\Uparrow}$ and B. D'Aguanno \\ $\dagger C R S 4, P S T$, Pula, Italy \\ $\ddagger$ School of Chemistry, University of Lincoln, Brayford Pool, LN6 7TS Lincoln, United \\ Kingdom \\ \Centre for Nanotechnology Research, VIT University, Vellore - 632014, Tamil Nadu, \\ India \\ E-mail: afloris@lincoln.ac.uk
}

December 9, 2020

\begin{abstract}
The specific heat behaviour in bulk nanomaterials (NMs) obtained by adding nanoparticles to pure suspending media has attracted a lot interest in recent years. Controversial results about NMs specific heat $\left(c_{p}\right)$ have been reported in literature, where nanoparticles (NPs) of different sizes and materials were suspended in solid and liquid salts, at different concentrations and temperatures. However, a unified picture explaining the $\mathrm{c}_{p}$ enhancements and diminutions by adding NPs to pure salts is still missing. In this work, we present a general theoretical thermostatic model aimed at describing the $\mathrm{c}_{p}$ behaviour in two-component ionic bulk nanomaterials containing NPs. The model, designed to work in the dilute regime, divides the NM in three regions: bulk suspending medium (SM), nanoparticles, and interface regions. It includes the effects of temperature, NPs size and NP concentration (mass fraction), allowing to calculate $c_{p}$ variations with respect to the pure SM and the ideal NM (where NP and SM are assumed to not interact). We then use the model
\end{abstract}


to interpret results of our classical molecular dynamics simulations, which we perform in the solid and liquid phases of NMs representative of three different classes, defined according to the atomic interactions at the interface. The analysis reveals non-trivial and competing effects influencing $\mathrm{c}_{p}$, such as system-dependent atomic rearrangements at the interface, vibrations of the $\mathrm{NP}$ as a whole and $\mathrm{c}_{p}$ variations coming from the individual NP and SM specific heats. Our study contributes to the interpretation of past controversial results and helps in designing NMs with improved thermal properties, which is highly relevant for industrial applications in thermal energy storage and renewable energy production.

\section{Keywords}

nanomaterials, classical molecular dynamics, specific heat, concentrated solar power, molten salts, thermal energy storage, renewable energy

\section{Introduction}

Using renewable energies is a key aspect in emerging issues such as climate change, greenhouse gas emissions and sustainable technologies. In this context, the exploitation of the thermal energy coming from the solar radiation plays a major role and it is heavily based on our ability to harvest, store and preserve this energy for a later use, when the solar radiation is weak or not available.

Among the technologies making use of thermal energy storage (TES), the Concentrated Solar Power (CSP) is the most important, reaching in 2019 a global capacity of $6.451 \mathrm{GW}$ with an annual growth rate of $6.29 \% .{ }^{1}$ Examples of TES systems include, e.g., the 125 MW Crescent Dunes Solar Energy CSP plant, which uses two TES tanks each able to hold 32 thousand tons of molten "solar salt", working at $\mathrm{T}=560 \mathrm{~K}$ and $\mathrm{T}=840$ $\mathrm{K}$, respectively. In industrial applications using such a large quantity of TES materials, even modest enhancements in the specific heat $\left(c_{p}\right)$ would have enormous consequences, as a drastic reduction of the tanks' size or, conversely, an increased quantity of stored 
energy.

Clearly, the capability of storing heat heavily relies on the chemical-physical properties of TES materials and improvements in their thermal properties are strongly required ${ }^{2}$ also in terms of costs per generated unit energy and TES capacities. ${ }^{1}$ The $c_{p}$ of a new generation of sensible TES materials should approach stretched target values of 3.7 - 4.5 $\mathrm{JK}^{-1} \mathrm{~g}^{-1},{ }^{3}$ an ambitious goal which seems, at the present, out of reach. For all these reasons, designing materials able to go beyond the thermal limits of known materials is imperative.

A promising paradigm generating a lot of expectations relies on the nanostructuring of bulk materials, to expand their thermal limits. ${ }^{4}$ Materials' nanostructuring allows to obtain nanomaterials (NMs) containing a huge amount of internal interface regions, whose thermodynamic properties could differ from the bulk. Table 1 illustrates the specific interface area values of some relevant systems. These are up to seven orders of magnitude larger than those of the corresponding bulk materials, a colossal increase. This could generate macroscopic, measurable effects, as for example, substantial $c_{p}$ enhancements.

Table 1: Specific interface area (in $\mathrm{cm}^{2} \mathrm{~g}^{-1}$ ) for foams, nanomaterials and bulk materials.

\begin{tabular}{|c|c|}
\hline Material & Specific interface area \\
\hline Microporous carbon foam & $\approx 2.9 \times 10^{7}$ \\
\hline Mesoporous carbon foam ${ }^{5}$ & $\approx 9.81 \times 10^{6}$ \\
\hline Macro-mesoporous carbon foam ${ }^{6,7}$ & $\approx 1.35-4.45 \times 10^{6}$ \\
\hline Natural graphite (porous) ${ }^{8}$ & $\approx 6.0 \times 10^{4}$ \\
\hline $\mathrm{SiO}_{2} \mathrm{NPs}_{\mathrm{s}}$ in $\mathrm{KNO}_{3}(5 \mathrm{~nm}, 20 \% \mathrm{wt})$ & $\approx 2.86 \times 10^{5}$ \\
\hline $\mathrm{SiO}_{2} \mathrm{NPS}_{\mathrm{s}}$ in $\left[\mathrm{C}_{4} \mathrm{mim}\right]\left[\mathrm{BF}_{4}\right]^{9}$ & $\approx 6.83 \times 10^{4}$ \\
\hline $\mathrm{KNO}_{3}$ & 4.71 \\
\hline Amorphous carbon & 3.66 \\
\hline Crystalline graphite & 3.12 \\
\hline $\mathrm{SiO}_{2}$ & 2.86 \\
\hline Diamond & 2.59 \\
\hline $\mathrm{Au}$ & $6.72 \times 10^{-1}$ \\
\hline
\end{tabular}

The $c_{p}$ behavior of NMs made by the inclusion of nanoparticles (NPs) in solid and liquid suspending media (SM) has attracted a huge interest in recent years. Investigations made by many research groups have been reviewed in several works, e.g. among others,

\footnotetext{
${ }^{1}$ The SunShot 2030 targets are to reach in ten years, a levelized cost of energy of $5 \mathrm{c} / \mathrm{kWh}$, with a TES capacity larger than 12 hours.
} 
by Arthur and Karim, ${ }^{10}$ Riazi et al. ${ }^{11,12}$ Hentschke $^{13}$ and, more recently, by Liu et al. ${ }^{14}$ After classifying the NMs according to their temperature regimes (from low/medium $T \simeq$ 300-450 K, to high $T \simeq 550-850 \mathrm{~K}$ ), these reviews try to obtain a consistent picture of the available experimental data and analyze the proposed mechanisms explaining the effects of NPs inclusion on the $c_{p}$ of liquid suspending media. However, the main conclusions of these studies are that $(i)$ the effect of NPs addition on the $c_{p}$ of the fluids does not yield a consistent picture ${ }^{10,13}$ and in molten salts causes anomalous enhancements/diminutions. (ii) the inconsistency depends on the base fluid, the NPs, and the synthesis method ${ }^{14}$ and there are even discrepancies among results of different studies using the same materials; ${ }^{12}$ (iii) a molecular theory explaining the effects of NPs on the $\mathrm{c}_{p}$ of heat transfer/storage fluids does not yet exist ${ }^{13,15}$ and the mechanisms driving the increase are not yet entirely understood. ${ }^{10,14}$

In this controversial scenario, the role of theoretical models and atomistic simulations to reach a unified picture of the various phenomena and their trends and to design nanomaterials with improved thermal properties, is crucial.

In this work, we develop a theoretical thermostatic model to describe the NMs $\mathrm{c}_{p}$ behaviour, where the NM is made by an ionic suspending medium with the inclusion of nanoparticles. We then perform classical molecular dynamics (MD) simulations to identify the mechanisms behind the $\mathrm{c}_{p}$ variation of several, qualitatively different NMs. The theoretical model is used to extrapolate MD results for nanoparticles inclusion of different sizes and mass fractions. To achieve a unified description, we investigate prototypical systems belonging to three classes of NMs: the first, characterized by cross interactions between the NP and the SM including both attractive/repulsive Coulomb potentials and van der Waals contributions; the second, where cross interactions are purely repulsive; and the third, in the solid phase, where nanoparticles are substituted by nano-holes, inducing atomic relaxations at the interface. The unified description is finally achieved by investigating both solid and liquid regimes, at four different temperatures. The combined analysis via the theoretical model and MD simulations allows us to define some design criteria of NMs. 
The paper is organized as follows: we first illustrate the theoretical model for the nanomaterials' specific heat. Next, we present MD and experimental results about the $\mathrm{c}_{p}$ of several pure constituents, namely different NPs and bulk $\mathrm{KNO}_{3}$. Then, we present our MD results for the $\mathrm{c}_{p}$ of amorphous silica NPs with silanol groups (a-SiO $\left.2 \mathrm{OH}\right)$, amorphous carbon NPs and nano-holes in solid $\mathrm{KNO}_{3}$. Next, we proceed with the MD study of the same NPs, but in the $\mathrm{KNO}_{3}$ liquid phase. Simulation results are presented in form of plots of $c_{p}$ enhancements with respect to the suspending medium and of density profiles which allow to analyse the atomic structures at the NP-SM interfaces. Results of the simulations are interpreted in the light of the proposed theoretical model. Finally, in the last section we draw our conclusions.

\section{Results and discussion}

There is a lack of a unified picture of the $c_{p}$ behaviour in NMs made of nanoparticles in solid and liquid salts. ${ }^{10-14}$ The latter materials are typically used in thermal energy storage industrial applications. ${ }^{16}$ From a fundamental point of view, it is important to understand the mechanisms of $\mathrm{c}_{p}$ enhancements/diminutions observed in available experiments, ${ }^{17-19}$ and to move from empirical ${ }^{20}$ towards more predictive models. ${ }^{21}$ From an applied perspective (e.g., to achieve $\mathrm{c}_{p}$ enhancements) it is crucial to arrive to define a set of criteria that NPs-SM combinations must fulfil.

In this work, we will consider different classes of systems, having the $\mathrm{KNO}_{3}$ salt as common SM. We expect that different salts, such as $\mathrm{NaNO}_{3}$ or the "solar salt" (60\% $\mathrm{NaNO}_{3}-40 \% \mathrm{KNO}_{3}$ ) used in industry, behave qualitatively in a similar way, due to their comparable microscopic structure. ${ }^{22,23}$ Each class is represented by a spherical nanoobject of different materials, with diameter $\sigma \approx 4.8-5.2 \mathrm{~nm}:(i)$ spherical amorphous silica NPs with silanol (Si-O-OH) surface groups, ${ }^{24,25}$ denoted a- $\mathrm{SiO}_{2} \mathrm{OH} ;(i i)$ spherical amorphous carbon (a-C) NPs and (iii) solid $\mathrm{KNO}_{3}$ in presence of nano-holes.

In systems containing silica, the NPs-SM interactions are a mix of attractive/repulsive Coulomb and van der Waals forces (see Supporting Information (SI) for details, Figure SI- 
1); in carbon systems, only repulsive interactions at the interface are present (Figure SI-2); in presence of nano-holes, $\mathrm{KNO}_{3}$ atoms are free re-organize, still changing the interface properties. The latter case is representative of systems as foams or solids containing empty cavities of different shapes. ${ }^{6,7}$ All these systems allow us to explore very different conditions at the interface, in solid and liquid regimes.

Molecular dynamics (MD) results for these nanomaterials need to be interpreted within a general picture. Therefore, in the following we present a theoretical thermostatic model of specific heat, for a two-components NMs, valid at any temperature and for any NP and SM material, in a dilute regime of NPs concentration (see below and SI for the precise definition of this regime).

\section{Theoretical thermostatic model for nanomaterials' specific heat}

The physical behavior of NMs made by two constituents is determined, to a large extent, by the formation of macroscopic interface regions between the constituents. As an exam-

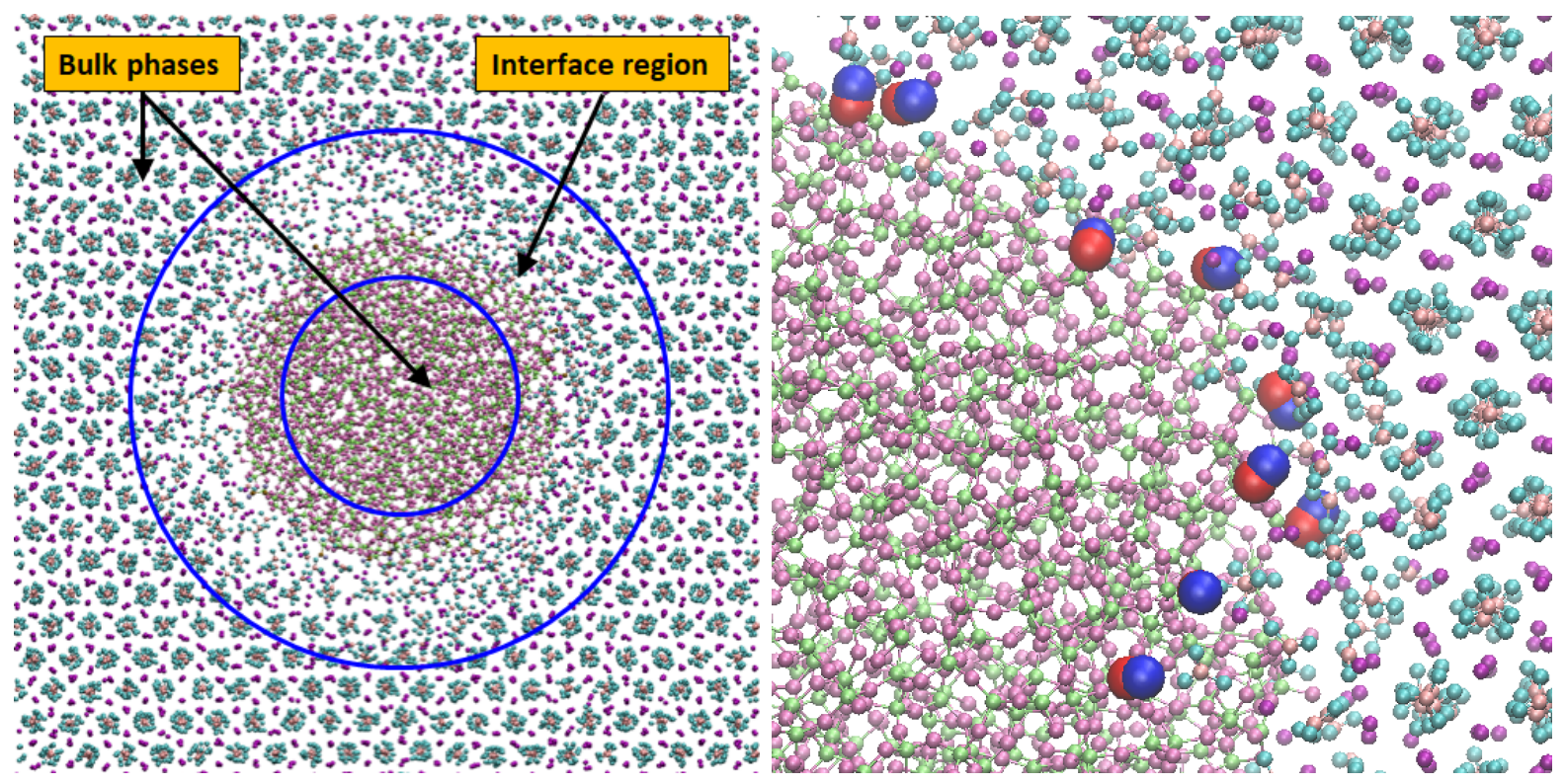

Figure 1: Left panel: snapshot of an amorphous a- $\mathrm{SiO}_{2} \mathrm{OH}$ nanoparticle in crystalline $\mathrm{KNO}_{3}$ at $T=550 \mathrm{~K}$. The bulk phases of the constituents and the interface region are indicated by arrows. Right panel: zoom of the interface region, with silanol groups highlighted. The two materials interpenetrate to a certain extent, as described in the main text. Color code: $\mathrm{K}$ (magenta), $\mathrm{N}$ (light brown), $\mathrm{O}_{\mathrm{KNO}_{3}}$ (cyan), Si (light green), $\mathrm{O}_{a-\mathrm{SiO}_{2} \mathrm{OH}}$ (pink). Selected -OH groups are highlighted in size in red-blue.

ple, in Figure 1 we show a snapshot of an a- $\mathrm{SiO}_{2} \mathrm{OH}$ spherical NP inside solid $\mathrm{KNO}_{3}$. Due 
to the interaction between the constituents, the NM structural and dynamical properties will differ from those of the constituents bulk phases.

A general theoretical model of the NM specific heat $\left(c_{P, n m}\right)$ can be developed by considering the NM as made of three subsystems: the NP, the bulk SM and the interface region (entirely made by SM particles). Considering NPs of diameter $\sigma$ in a SM at the temperature $T$, we will derive a general expression for $c_{P, n m}(T, \sigma)$ assumimg, for simplicity, spherical NPs ${ }^{2}$. First, we give the expression of the NM specific heat $c_{P, n m}^{i d}(T, \sigma)$ in the ideal case, i.e. neglecting the interaction between the constituents:

$$
c_{P, n m}^{i d}(T, \sigma)=x_{n p} c_{P, n p}(T, \sigma)+x_{s m} c_{P, s m}(T) .
$$

Here, $c_{P, n p}(T, \sigma)$ and $c_{P, s m}(T)$ are the specific heats of the NP and the bulk SM, respectively, and $x_{n p}=\frac{m_{n p}}{m_{t}}$ and $x_{s m}=\frac{m_{s m}}{m_{t}}$ are the mass fractions of the NM total mass $m_{t}$, where $m_{n p}$ and $m_{s m}$ are the NPs and the SM total masses in the system $\left(x_{n p}+x_{s m}=1\right)$. Due to the NP-SM interaction, $c_{P, n m}(T, \sigma)$ will in general deviate from its ideal counterpart $c_{P, n m}^{i d}(T, \sigma)$. The specific heat $c_{P, n m}(T, \sigma)$ can always be decomposed as:

$$
c_{P, n m}(T, \sigma)=c_{P, n m}^{i d}(T, \sigma)+c_{P, e x}(T, \sigma)
$$

where we implicitly define the excess term $c_{P, e x}(T, \sigma)$ as the difference between the NM physical (fully interacting) specific heat and its ideal (non interacting) counterpart. This term is central in our analysis as it incorporates all the contributions (thermal degrees of freedom) to $c_{p}$ generated by the interaction between the constituents ${ }^{3}$. In order to find its analytical expression, we will make the assumption that the NP surface is only slightly modified by the interaction, while the SM structure can be strongly affected by the presence of the NPs. The subsystems have the mass fractions $x_{n p}$ (for the NP), $x_{s m, b}$ (for the SM bulk) and $x_{s m, i n t}$ (for the SM interface region), where $x_{s m, b}+x_{s m, i n t}=x_{s m}$. Correspondingly, each subsystem has an associated specific heat: $c_{P, n p}(T, \sigma), c_{P, s m}(T)$ and $c_{P, \text { int }}(T, \sigma)$. Hence, $c_{P, n m}(T, \sigma)$ can be expressed as:

\footnotetext{
${ }^{2}$ The extension to other NPs geometries is straightforward.

${ }^{3}$ Note that the excess term $c_{P, e x}(T, \sigma)$ can be also a negative quantity.
} 


$$
c_{P, n m}(T, \sigma)=x_{n p} c_{P, n p}(T, \sigma)+x_{s m, b}(T, \sigma) c_{P, s m}(T)+x_{s m, i n t}(T, \sigma) c_{P, i n t}(T, \sigma) .
$$

From Eqs. (2) and (3), we have:

$$
c_{P, e x}(T, \sigma)=x_{s m, i n t}(T, \sigma)\left(c_{P, \text { int }}(T, \sigma)-c_{P, s m}(T)\right)
$$

Eqs. (2) and (4) show that due to the NP-SM interaction, it is possible to obtain nanomaterials with a $c_{P, n m}(T, \sigma)$ higher or lower than in the ideal mixture, depending whether the $c_{P, \text { int }}(T, \sigma)$ of the interface region is larger or smaller than $c_{P, s m}(T)$, associated to the SM bulk. In this way the specific heat enhancement $c_{P, n m}(T, \sigma)-c_{P, n m}^{i d}(T, \sigma)$ is defined as a variation with respect to ideal value. Alternatively, the enhancement can be also given in percentage with respect to $c_{P, s m}(T)$, as $\eta(T, \sigma)=c_{P, n m}(T, \sigma) / c_{P, s m}(T)-1$, as typically done in the experimental literature. Here, results will be analysed by using both definitions. According to Eqs. (1) and (2), we can obtain NMs with a $c_{P, n m}(T, \sigma)$ higher or lower than $c_{P, s m}(T)$ under the conditions:

$$
c_{P, n m}(T, \sigma)-c_{P, s m}(T) \gtreqless 0 \quad \longrightarrow \quad c_{P, n p}(T, \sigma)+\frac{c_{P, e x}(T, \sigma)}{x_{n p}} \gtreqless c_{P, s m}(T) .
$$

Clearly, Eq. (5) shows that if there is no significant interaction between the constituents $\left(c_{P, e x}(T, \sigma) \approx 0\right)$, the specific heat of the nanomaterial will approach the ideal value and will increase (decrease) as soon as we add nanoparticles whose specific heat is higher (lower) than $c_{P, s m}(T)$, for any finite NP mass fraction (see also Eq. 2).

The experimental data of $c_{P, n m}(T, \sigma)$, as obtained for a variety of NPs and SMs, ${ }^{10-14}$ show that $c_{P, n m}(T, \sigma)$ strongly deviates from the ideal behavior, but the data interpretation is often controversial and rarely based on the systematic dependence of $c_{P, n p}(T, \sigma)$ and $c_{P, s m}(T)$ on temperature and NP size. In this complex experimental scenario, the excess term $c_{P, e x}(T, \sigma)$ plays an essential role and incorporates the microscopic mechanisms controlling the $c_{P, n m}(T, \sigma)$ variation due to the interaction of the constituents.

Eq. (4) is particularly relevant in the dilute regime. We define this regime for NPs dispersions as the mass fraction interval $x_{n p} \in\left[0, x_{n p}^{\text {dilute }}\right]$, where the $c_{P, n m}\left(x_{n p}\right)$ behaviour 
is linear and approximates well the experimental specific heat of the dispersions, in the exact, same spirit of the Henry's law for solutions. ${ }^{26-29}$ (see also SI for a full discussion). In this regime, the atomic structure of the interface region in presence of many NPs is the same as it would be if there was a single NP in the entire system, implying that $c_{P, \text { int }}(T, \sigma)$ (a quantity per unit mass) will not depend on the NPs mass fraction or number concentration. ${ }^{4}$ For spherical NPs in the dilute regime, the mass fraction of the interface region in Eq. (4) reads (see SI for the derivation):

$$
x_{s m, i n t}(T, \sigma)=x_{n p} \frac{\rho_{\text {sm,int }}(T, \sigma)}{\rho_{1 p}(T)}\left[\left(1+2 \frac{\delta_{\text {int }}(T, \sigma)}{\sigma}\right)^{3}-1\right]
$$

where $\rho_{s m, i n t}(T, \sigma)$ is the density of the interface and $\rho_{1 p}(T)$ is the density of one NP. ${ }^{5}$ Eq. (6) (entering Eqs. (4) and (2)) shows that, for example, for a positive specific heat enhancement $\left(c_{P, e x}(T, \sigma)>0\right)$, the latter increases when the NP weight fraction $x_{n p}$ increases. Moreover, it will increase when the NP diameter $\sigma$ decreases and when the NP density $\rho_{1 p}(T)$ decreases: lighter and smaller NPs will generate larger deviations from the ideal behavior. This fact can be explained by noticing that at a given $x_{n p}$ and $\rho_{1 p}(T)$, a reduction of $\sigma$ implies an increase in the NPs number concentration, i.e. an increase of the total interface volume. Likewise, at fixed $x_{n p}$ and $\sigma$, lighter NPs again imply a larger NPs number concentration and total interface volume. Clearly, this volume depends also on the thickness $\delta_{i n t}(T)$, whose value can be extracted by the system density profiles obtained via a MD simulations (see below), even if, strictly speaking, its determination is not extremely precise. Several methods have been proposed in the literature, ${ }^{30,31}$ here we used the one of Ref., ${ }^{31}$ based on the systematic deviations of the density from the average bulk density.

The generality of this model can be fully exploited by combining it with MD calculations of $c_{P, n m}(T, \sigma), c_{P, n p}(T, \sigma)$ and $c_{P, s m}(T)$ performed at a given $T, \sigma$ and $x_{n p}$, for the respective three systems. Once their values are known, Eq. (1) allows to obtain

\footnotetext{
${ }^{4}$ In a non-dilute regime, the interfaces overlap would affect their structures, and $c_{P, \text { int }}(T, \sigma)$ could depend on $x_{n p}$ and number concentration (depending on $d, \sigma, \delta_{i n t}$ and on how many NPs are involved in the interface "merging").

${ }^{5}$ For small variations of $\sigma, \rho_{\text {sm,int }}(T, \sigma)$ and $\delta_{\text {int }}(T, \sigma)$ are assumed to be $\sigma$ independent, i.e. $\rho_{\text {sm,int }}(T, \sigma) \approx \rho_{\text {sm,int }}(T)$ and $\delta_{\text {int }}(T, \sigma) \approx \delta_{\text {int }}(T)$.
} 
$c_{P, n m}^{i d}(T, \sigma)$, which in turn makes possible to extract the excess term $c_{P, e x}(T, \sigma)$ from Eq. (2). Next, $c_{P, i n t}(T, \sigma)$ can be obtained from Eqs. (6) and (4). As $c_{P, i n t}(T, \sigma)$ in the dilute regime is independent of $x_{n p}$, its knowledge at $(T, \sigma)$ allows to obtain $c_{P, e x}(T, \sigma)$ and finally the specifc heat of the nanomaterial $c_{P, n m}(T, \sigma)$, at any $x_{n p}$ in the whole dilute regime at a given $(T, \sigma)$. The excess term $c_{P, e x}(T, \sigma)$ will be a linear function of $x_{n p}$ (from Eqs. 6 and 4).

The theoretical model allows also to avoid MD calculations at different NP diameters $\sigma$. From enthalpy considerations it is possible to write $c_{P, e x}(T, \sigma)$ as:

$$
c_{P, e x}(T, \sigma)=\frac{A}{m_{t}} C_{A, e x}(T),
$$

where $C_{A, e x}(T)$ is an excess term per nanoparticle unit surface, $A$ is the sum of the surface areas of all NPs and $m_{t}$ is the total mass of the system. For spherical NPs, Eq. (7) reads (see SI for the derivation of Eqs. 7 and 8):

$$
c_{P, e x}(T, \sigma)=x_{n p} \frac{6}{\sigma \rho_{1 p}(T)} C_{A, e x}(T) .
$$

The excess term $C_{A, e x}(T)$ is a relevant, fundamental quantity, as it depends only on the nature of the materials at the interface. In fact we are assuming, very reasonably, that for small variations of $\sigma, C_{A, e x}(T)$ is independent on the NP surface curvature, hence $\sigma$ appears only as a prefactor in Eq. (8). ${ }^{6}$

In summary, the independence of $C_{A, e x}(T)$ of $\sigma$ allows to know the excess term $c_{P, e x}(T, \sigma)$ (and then specific heat enhancements illustrated in the next Sections) at any $x_{n p}$, and $\sigma$ compatible with the dilute regime, via a set of MD simulations for the three systems (NM, NP and bulk SM) performed at given $(T, \sigma)$. In the SI a quantitative analysis is made, to set the limits that a dilute regime imposes to $x_{n p}$ values. Our model then allows to have information about the specific heat of the NMs (avoiding extra MD calculations) in very large intervals of $x_{n p}$ and $\sigma$, while only a temperature change will require new MD simulations, as will be illustrated below.

\footnotetext{
${ }^{6}$ Note that the trends of increasing/decreasing excess terms from Eq. (8) are similar to the ones from Eqs. (4) and (6).
} 


\section{Specific heat of constituents and NMs ideal behaviour}

We will now calculate, via MD, the specific heat of the constituents analyzed in this work. Figure 2 shows the $c_{P, s m}(T)$ values of bulk $\mathrm{KNO}_{3}$ as a function of temperature,

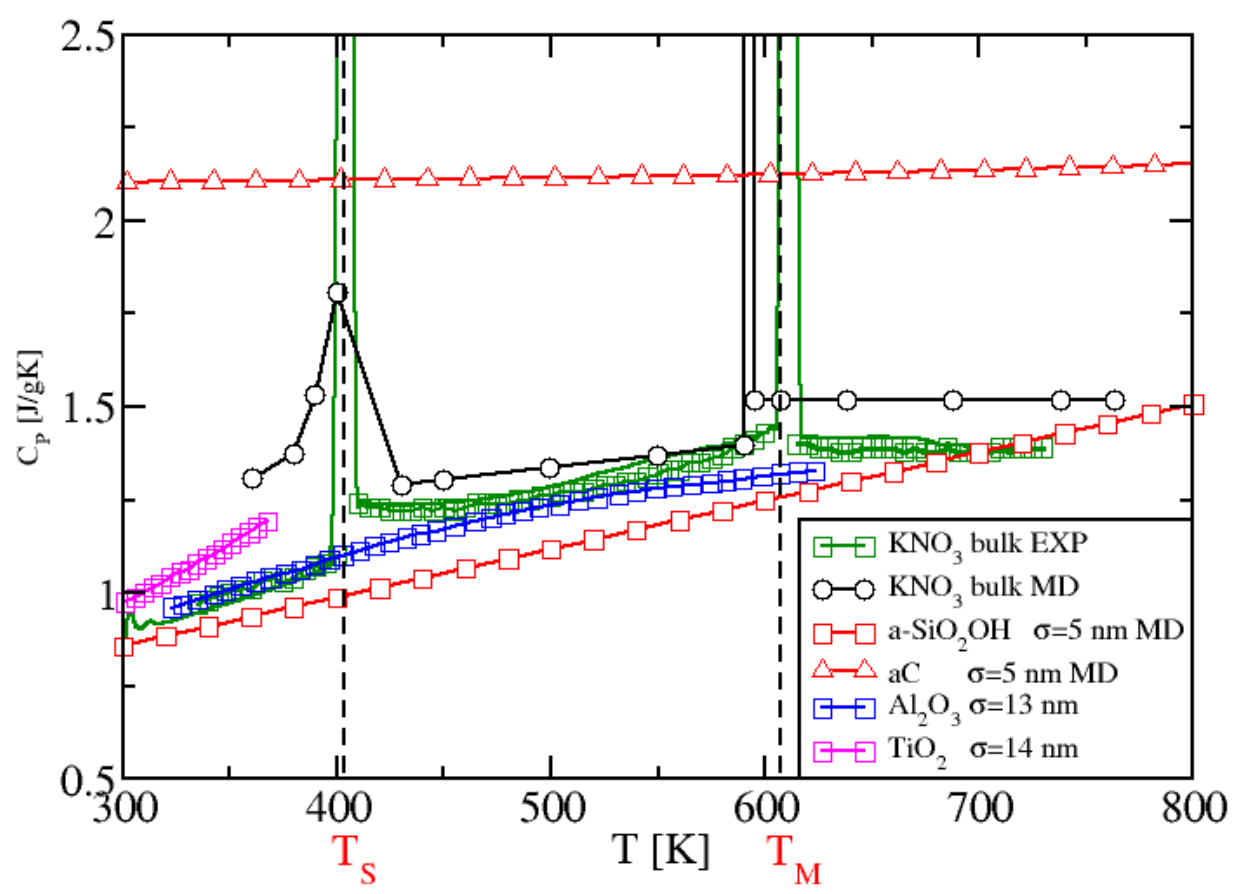

Figure 2: Specific heat of bulk $\mathrm{KNO}_{3}$ from room temperature to its thermal stability limit, in its solid and liquid phase (experimental ${ }^{32,33}$ and calculated via $\mathrm{MD}^{22}$ ). $\mathrm{T}_{S}$ and $\mathrm{T}_{M}$ indicate solid-to-solid and solid-to-liquid experimental transition temperatures of the material. We show also the $c_{P, n p}(T)$ of different nanoparticles: a- $\mathrm{SiO}_{2} \mathrm{OH}$ and a-C (calculated in this work); $\mathrm{Al}_{2} \mathrm{O}_{3}{ }^{32}$ and $\mathrm{TiO}_{2} \cdot{ }^{33}$

together with MD calculated $c_{P, n p}(T, \sigma)$ of a-SiO${ }_{2} \mathrm{OH}$ and a-C NPs and experimental values of $\mathrm{Al}_{2} \mathrm{O}_{3}$ and $\mathrm{TiO}_{2}$ NPs. The NMs specific heat ideal behaviour when adding NPs of different materials to bulk $\mathrm{KNO}_{3}$, can be analyzed via Eq. (1). Adding a$\mathrm{SiO}_{2} \mathrm{OH}$ NPs implies $c_{P, n m}^{i d}<c_{P, s m}$, as $c_{P, n p}<c_{P, s m}$ (except for $T \gtrsim 750 \mathrm{~K}$, where MD calculations give $\left.c_{P, n m}^{i d}>c_{P, s m}\right)$. Adding a-C NPs produces $c_{P, n m}^{i d}>c_{P, s m}$ for all considered temperatures. The same should happen for $\mathrm{TiO}_{2}$ NPs. Finally, adding $\mathrm{Al}_{2} \mathrm{O}_{3}$ NPs produces a $c_{P, n m}^{i d} \simeq c_{P, s m}$. These preliminary considerations will play a role in the analyses of the $c_{p}$ variations in the fully interacting NMs, with respect to the SM, which we will present below for solid and liquid systems. 


\section{Specific heat of solid nanomaterials}

In this Section, we will discuss the properties of solid NMs. Combining results of MD simulations and of the theoretical model illustrated above, we will analyze the behaviour of different systems leading to different $c_{p}$ enhancements/diminutions.

a-SiO ${ }_{2} \mathbf{O H}$ nanoparticles in solid $\mathbf{K N O}_{3}$. We first consider a-SiO${ }_{2} \mathrm{OH}$ NPs $(\sigma=5.2$ $\mathrm{nm}$ ) in solid potassium nitrate. The NPs have silanol (Si-O-OH) surface groups at the experimental silanol concentrations ${ }^{34}$ (Figure 1, right panel).

Experimentally, $\mathrm{KNO}_{3}$ undergoes a solid-to-solid structural transition at $\mathrm{T}_{S}=403$ $\mathrm{K}$ and a solid-to-liquid transition at $\mathrm{T}_{M}=607 \mathrm{~K}$ (see Figure 2). ${ }^{22,35}$ We simulate the system at 450 and $550 \mathrm{~K}$, at ambient pressure $(P=1 \mathrm{~atm})$. These temperatures have been chosen between $\mathrm{T}_{S}$ and $\mathrm{T}_{M}$, where the experimental and simulated $\mathrm{c}_{p}$ data for pure $\mathrm{KNO}_{3}$ are very stable. The MD calculated specific heats are summarized in Table Table 2: Calculated $\mathrm{c}_{p}$ for solid nanomaterials, using $\mathrm{KNO}_{3}$ as suspending medium. NPs and nano-holes have diameter $\sigma=4.8-5.2 \mathrm{~nm}$. Temperatures $(\mathrm{T})$ are given in $\mathrm{K}, \mathrm{c}_{p}$ 's in $\mathrm{JK}^{-1} \mathrm{~g}^{-1}$ and $C_{A, e x}$ in $10^{-7} \times \mathrm{JK}^{-1} \mathrm{~g}^{-1} \mathrm{~cm}^{-2}$. Note that the $x_{n p}$ 's for a- $\mathrm{SiO}_{2} \mathrm{OH}$ and a-C are slightly different, so that the $c_{p, e x}$ 's are not immediately comparable (they depend on $x_{n p}$, Eq. 8), while $C_{A, e x}$ 's are (they do not depend on mass fractions).

\begin{tabular}{|c|c|c|c|c|c|c|c|c|}
\hline & $\mathrm{T}$ & $c_{P, n p}$ & $c_{P, s m}$ & $x_{n p}$ & $c_{P, n m}^{i d}$ & $c_{P, n m}$ & $c_{P, e x}$ & $C_{A, e x}$ \\
\hline \multirow{2}{*}{$\begin{array}{c}\mathrm{a}_{-} \mathrm{SiO}_{2} \mathrm{OH} \\
\mathrm{NP}\end{array}$} & 450 & 1.052 & 1.303 & 0.0638 & 1.287 & 1.360 & 0.073 & 2.27 \\
\cline { 2 - 9 } & 550 & 1.182 & 1.369 & 0.0638 & 1.357 & 1.417 & 0.060 & 1.86 \\
\hline $\mathrm{a}-\mathrm{C}$ & 450 & 2.113 & 1.303 & 0.0408 & 1.347 & 1.492 & 0.156 & 5.77 \\
\cline { 2 - 9 } $\mathrm{NP}$ & 550 & 2.120 & 1.369 & 0.0408 & 1.410 & 1.658 & 0.258 & 9.55 \\
\hline $\begin{array}{c}\text { Nano } \\
\text { hole }\end{array}$ & 450 & - & 1.303 & - & 1.303 & 1.326 & 0.023 & 1.5 \\
\cline { 2 - 9 } & 550 & - & 1.369 & - & 1.369 & 1.396 & 0.027 & 1.65 \\
\hline
\end{tabular}

2. The $\mathrm{c}_{P, n m}$ values are 1.360 and $1.417 \mathrm{JK}^{-1} \mathrm{~g}^{-1}$ at 450 and $550 \mathrm{~K}$, respectively. At the same temperatures, $c_{P, s m}$ of $\mathrm{KNO}_{3}$ is 1.303 and 1.369 , and $\mathrm{c}_{P, n m}^{i d} 1.287$ and 1.357 . As the nanoparticle $\mathrm{c}_{P, n p}$ 's are lower than $\mathrm{c}_{P, s m}$, simply adding a-SiO${ }_{2} \mathrm{OH}$ NPs to solid $\mathrm{KNO}_{3}$ within an ideal model can only decrease $c_{P, n m}^{i d}$ and the reduction is more substantial the larger the NP mass fraction (Eq. 1). Crucially instead, at $450 \mathrm{~K}$ and $x_{n p}=0.0638$ the specific heat $c_{P, n m}$ is higher than $c_{P, s m}$ (by $4.4 \%$ ) and than $c_{P, n m}^{i d}$ (by $5.7 \%$ ). This means that the interaction is not only able to counterbalance the lower $\mathrm{c}_{P, n p}$ of the nanoparticles, but also to revert the trend, producing a positive enhancement. 


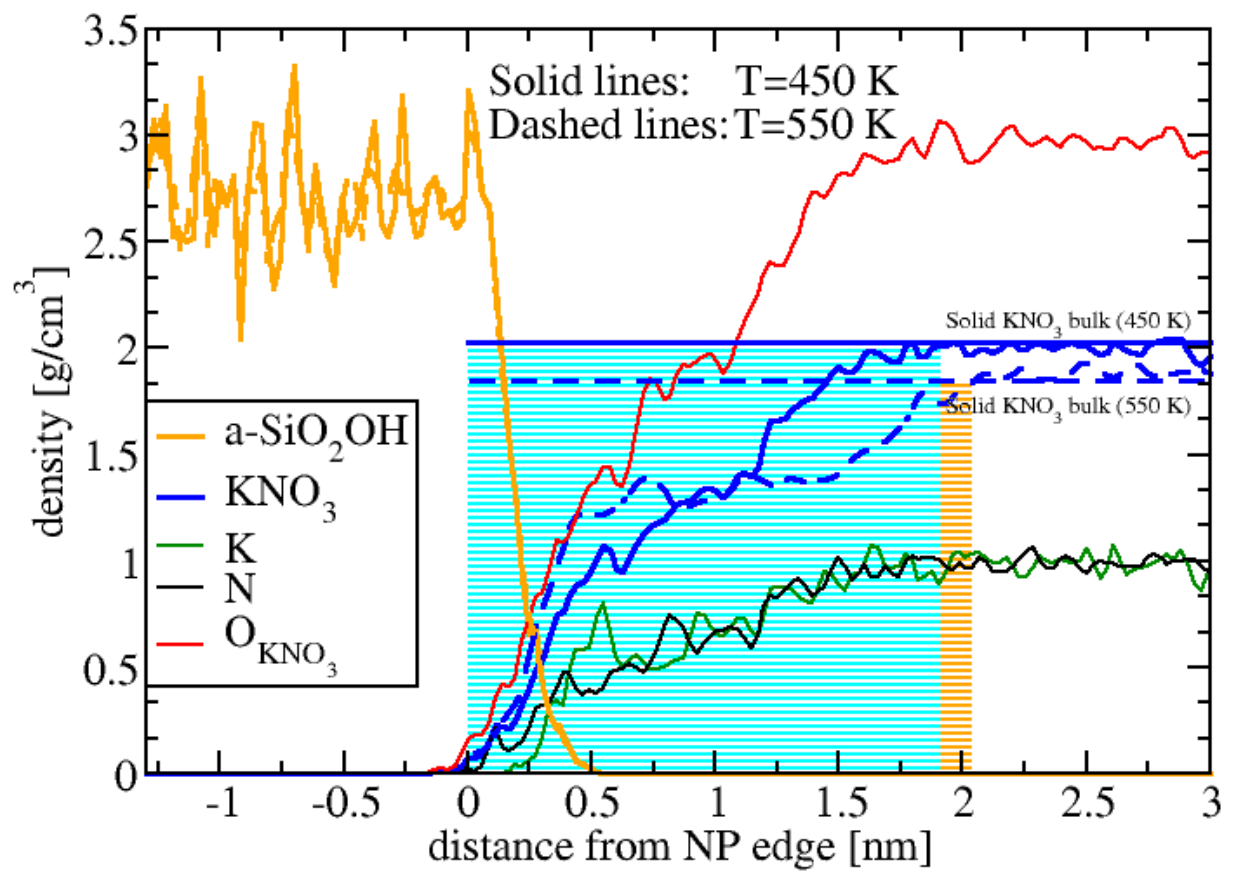

Figure 3: Density profiles of solid $\mathrm{KNO}_{3}$ (total and from individual atoms) around an a$\mathrm{SiO}_{2} \mathrm{OH}$ nanoparticle at $T=450$ and $550 \mathrm{~K}$. Blue horizontal lines are constant densities in the $\mathrm{KNO}_{3}$ bulk. The density of the NP silanol groups is not shown. Densities of individual atoms are shown for $T=450 \mathrm{~K}$ only and are in adimensional units, normalized to their stoichiometry in the $\mathrm{KNO}_{3}$ bulk. The zero of the $x$ axis is located at the edge of the NP, to emphasize the thickness of the interface regions, highlighted in cyan and orange for the two temperatures. 
To understand the microscopic mechanisms leading to this increase, in Figure 3 we show the density profiles of the interface region at 450 and $550 \mathrm{~K}$. We define ${ }^{31}$ the $\mathrm{KNO}_{3}$ interface thickness $\delta_{\text {int }}$ as the distance from the NP surface density peak (at 0 $\mathrm{nm})$ to the region where the bulk $\mathrm{KNO}_{3}$ density and structure are recovered. At $450 \mathrm{~K}$ this happens at $\approx 1.9 \mathrm{~nm}$, thus $\delta_{\text {int }} \approx 1.9 \mathrm{~nm}$ (see highlighted interface region in cyan). At $550 \mathrm{~K}$ the thickness slightly increases to $\delta_{\text {int }} \approx 2.0 \mathrm{~nm}$ (highlighted in orange), with the a- $\mathrm{SiO}_{2} \mathrm{OH}$ density virtually unchanged. These density profiles stem from the cross interaction potential terms between the NP and the $\mathrm{KNO}_{3}$ atoms (shown in Figure SI-1). In turn, the terms result from attractive van der Waals and attractive/repulsive Coulomb forces, which depend on the specific atoms considered. Inside the interface region, the density profile of solid $\mathrm{KNO}_{3}$ clearly shows a depletion with respect to the solid bulk. Interestingly, the profiles show a region (between $\approx 0$ and $0.56 \mathrm{~nm}$ ) where the NP and SM interpenetrate (see also Figure 1). We anticipate that in the liquid regime, a different behavior is obtained (see Figure 10) as, beyond the interpenetrating region, the liquid $\mathrm{KNO}_{3}$ density "piles up" at the interface, indicating an effective NP-SM net attraction. The lack of pile-up in the solid is due to the large cohesive energy of the solid phase, where the $\mathrm{KNO}_{3}$ atoms at the interface feel a stronger attraction to the solid bulk than to the nanoparticle's atoms. In the liquid, the bonding inside the bulk is weaker, thus the NP-SM attraction prevails.

The $c_{p}$ enhancement implies an excess term $c_{P, e x}(T, \sigma)$ larger than zero. Likewise, the purely material's dependent $C_{P, e x}(T)$ term is positive (see Eq. 7 and Table 2). From Eq. (4), this means that the interface specific heat $c_{P, \text { int }}(T, \sigma)$ is larger than in the bulk $\left(c_{P, s m}(T)\right)$, toward values typical of a liquid phase. In summary, the interface atoms of solid $\mathrm{KNO}_{3}$ behave as they were in a frozen "liquid-like" state, less dense than the bulk and characterized by much softer vibrational modes, thus more prone to absorb energy and with a larger specific heat. The existence of a liquid-like layer in crystalline finite system (i.e. containing a surface) has been observed also in Refs., ${ }^{31,36,37}$ which also show that the $c_{p}$ of the crystalline finite system is larger than the one of the corresponding bulk (without surface). 


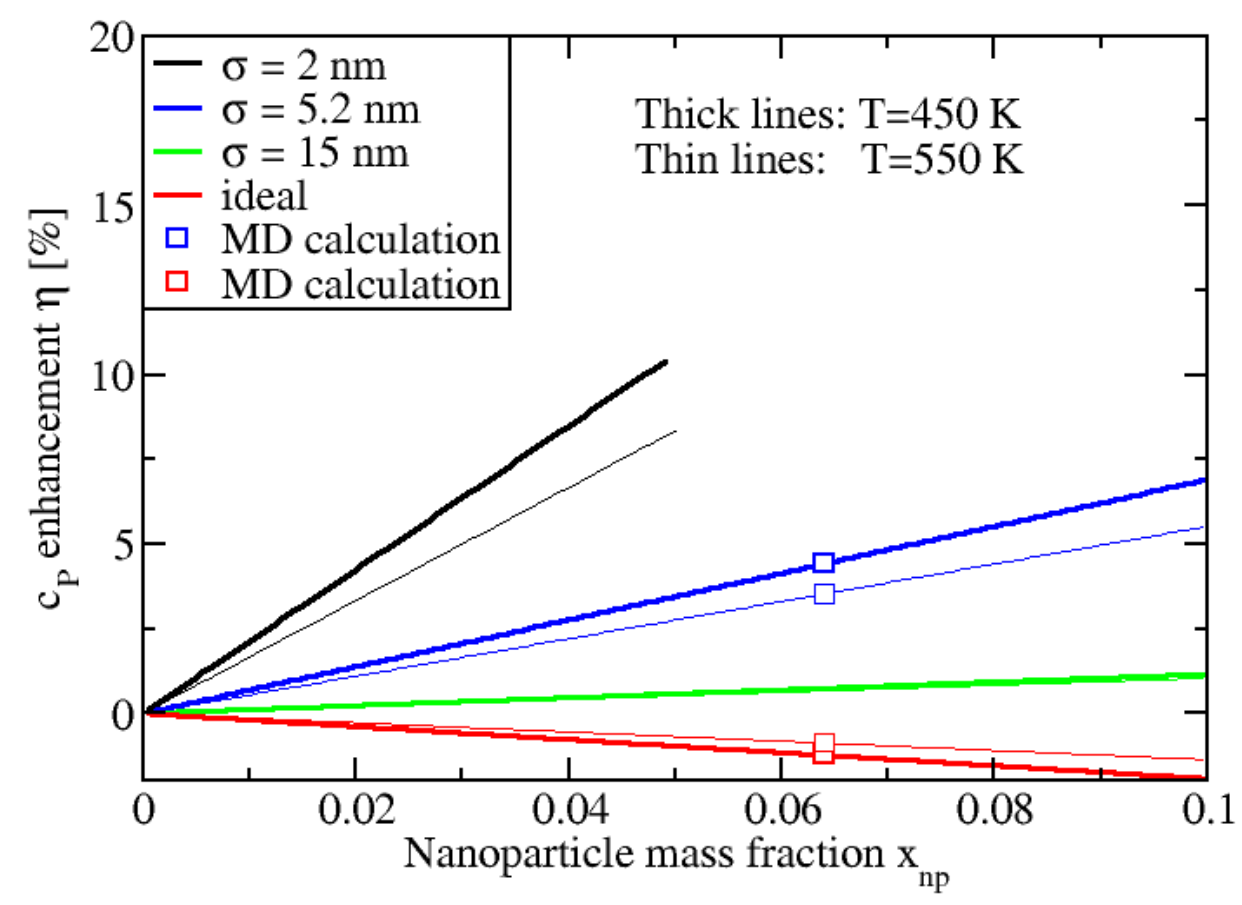

Figure 4: Specific heat enhancement $\eta=c_{P, n m} / c_{P, s m}-1$ (in percent, relative to the suspending medium) of a- $\mathrm{SiO}_{2} \mathrm{OH}$ nanoparticles in solid $\mathrm{KNO}_{3}$ as function of the NP's diameter $\sigma$ and mass fraction $x_{n p}$, at $T=450$ and $550 \mathrm{~K}$. Lines are obtained by our theoretical model, empty symbols refer to MD simulated systems. Blue symbols indicate the enhancement of the fully interacting system, while red symbols the one of the corresponding ideal system. Plots are truncated for mass fraction values where the nanomaterial cannot be considered in the dilute regime (see SI for details and Table SI-7). 
We can now use our theoretical model to obtain the specific heat enhancements for different NP mass fractions and diameters, without performing extra MD simulations. Figure 4 illustrates the $\mathrm{c}_{p}$ enhancement $\eta$ in the interval $x_{n p} \sim 0$ till $\sim 0.1$. Here, the system is estimated to be in a dilute regime for several nanoparticle sizes, apart from $\sigma=2 \mathrm{~nm}$, where the maximum mass fraction compatible with the dilute regime is $x_{n p} \sim 0.05$. Quantitative criteria to characterize this regime are discussed in SI and Tables SI-7 and SI-8. Figure 4 highlights the opposite behaviour of $\mathrm{c}_{P, n m}$ and $\mathrm{c}_{P, n m}^{i d}$. Importantly, $\mathrm{c}_{P, n m}$ increases with the NP mass fraction. We note also that: $(i)$ smaller NPs allow a much stronger enhancement than larger ones (for $\sigma=15 \mathrm{~nm}$ there is hardly any enhancement); (ii) smaller NPs have a much faster rate of change of $\eta$ with increasing $x_{n p}$; (iii) the $\mathrm{c}_{P, n m}$ of NMs including smaller NPs is, however, more sensitive to temperature changes, with less pronounced enhancements at higher temperatures.

Quantitatively, the most striking result of Figure 4 is the $10 \%$ specific heat enhancement at $x_{n p}=0.05$ for a $\sigma=2 \mathrm{~nm} \mathrm{NP}$ at $T=450 \mathrm{~K}$.

Positive enhancements in experimental investigations have been reported for silica and alumina NPs in solid nitrates, ${ }^{38-41}$ in line with our results.

Carbon nanoparticles in solid $\mathbf{K N O}_{3}$. We now turn to a different system, made of amorphous carbon nanoparticles (a-C) in solid $\mathrm{KNO}_{3}$. Here, we find that the cross terms of the interaction are purely repulsive, as can be seen in Figure SI-2. The corresponding density profiles are shown in Figure 6. This system is qualitatively different from the previous one, both in the solid and liquid phase. The repulsion is such that the thickness of the $\mathrm{KNO}_{3}$ interface region is rather small, $\delta_{\text {int }} \approx 0.86 \mathrm{~nm}$ at $450 \mathrm{~K}$ and $\approx 1.0 \mathrm{~nm}$ at $550 \mathrm{~K}$ (see cyan and orange regions in Figure 6 ). These values are $\approx 1 \mathrm{~nm}$ smaller than in the a-SiO 2 OH NPs case. Moreover, the a-C NP density goes rapidly to zero (the nanoparticle depletion region is $\approx 0.3 \mathrm{~nm}$ smaller than in the $\mathrm{a}-\mathrm{SiO}_{2} \mathrm{OH}$ case). As a result, the densities associated to the a-C NP and $\mathrm{KNO}_{3}$ do not interpenetrate. With these findings, one can be tempted to assign to this system an ideal behavior. However, this is by far not the case. Due to the nanoparticles inclusion, the nanomaterial exhibits a strong $c_{p}$ enhancement with respect to the pure salt, much larger than in the a- $\mathrm{SiO}_{2} \mathrm{OH} \mathrm{NP}$ case, 
as shown in Figure 7. The entity of the enhancement $\eta$ is due to several reasons, which can be understood via our theoretical model and the density profiles. First, unlike with a- $\mathrm{SiO}_{2} \mathrm{OH}$ NPs, here even the ideal term $c_{P, n m}^{i d}$ gives a positive contribution to $\eta$ (Figure 7 ), as $c_{P, n p}$ is much higher than $c_{P, s m}$ (Figure 2). Second, the interface region gives a positive contribution to $\eta$, even if by a smaller entity (per NP) as compared to the a$\mathrm{SiO}_{2} \mathrm{OH}$ case, where the interface region volume is larger. A third contribution comes from the purely repulsive interactions between the two nanomaterial's components (Figure SI2). This implies the presence of an excluded volume region around the a-C nanoparticle, indicated by the black arrows in Figure 6. This region, not present with a- $\mathrm{SiO}_{2} \mathrm{OH}$ NPs, allows the a-C NPs the possibility to have a strong vibrational motion as a whole, as can be inferred from the a-C NP center of mass displacements shown in Figure 5 (right panel), with a-C NPs vibration amplitudes doubled as compared to the a- $\mathrm{SiO}_{2} \mathrm{OH}$ case, Figure 5 (left panel). This contribution is not explicitly described in our (thermostatic) theoretical model, but is fully taken into account in the MD simulations results (i.e. in the $c_{P, e x}$ and $C_{A, e x}$ terms), which, together with the model, determine the $x_{n p}$ and $\sigma$ extrapolations shown in Figure 7. Note that these second and third contributions are,
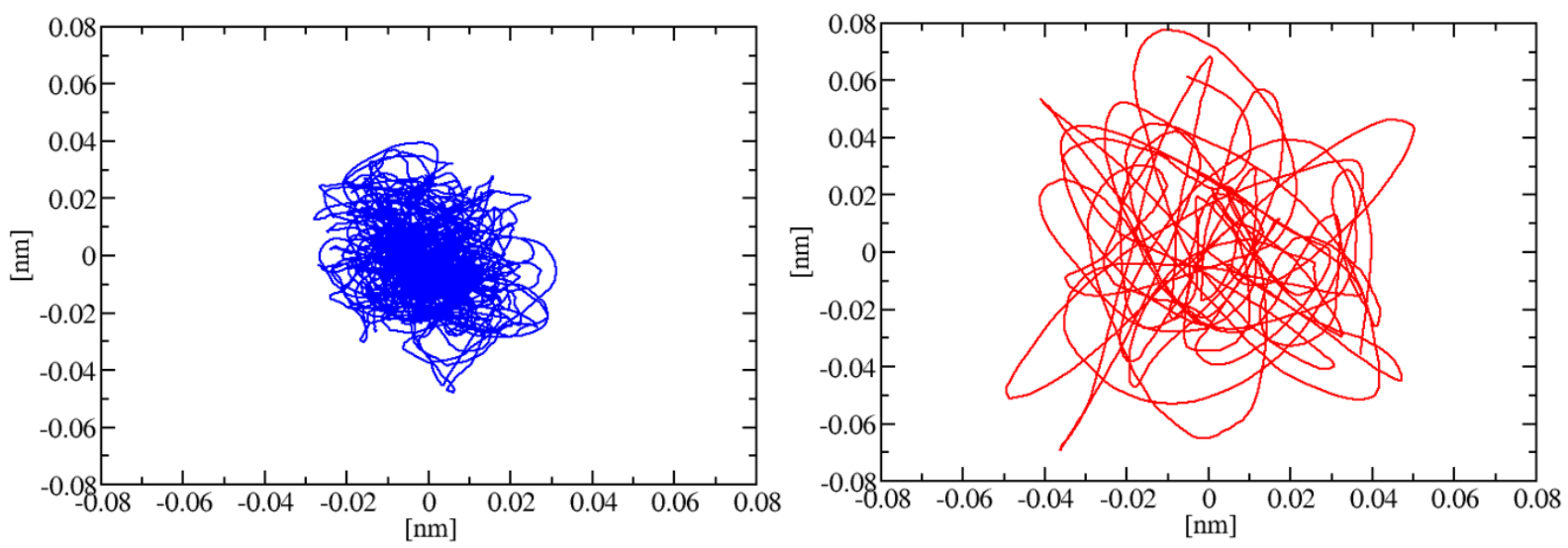

Figure 5: Center of mass trajectories (vibrations) of a- $\mathrm{SiO}_{2} \mathrm{OH}$ (left panel) and a-C (right panel) nanoparticles in solid $\mathrm{KNO}_{3}$, at $T=550 \mathrm{~K}$. The a-C NP vibration amplitude is roughly twice as large as in the a- $\mathrm{SiO}_{2} \mathrm{OH}$ case.

ultimately, related only to the repulsive nature of the materials at the interface. Thus, in Table 2 we see that the $C_{A, e x}$ values for a-C in solid $\mathrm{KNO}_{3}$, are much larger than in the a- $\mathrm{SiO}_{2} \mathrm{OH}$ case. A fourth, crucial reason to explain the large $c_{p}$ enhancement is the fact that a-C nanoparticles are light. They have a small mass density $\left(\rho_{1 p} \approx 1.89 \mathrm{~g} \mathrm{~cm}^{-3}\right)$ as 
compared to a-SiO ${ }_{2} \mathrm{OH}$ NPs $\left(\rho_{1 p} \approx 2.26 \mathrm{~g} \mathrm{~cm}^{-3}\right)$ (see Table SI-9). The effect of having light NPs is illustrated in Eq. (8) of our model. Importantly, at a given mass fraction $x_{n p}$ and diameter $\sigma$, a small NP mass density implies an increased number concentration of nanoparticles, thus amplifying the enhancements due to the previous effects.

A final aspect in the comparison between the two systems is the different behaviour of $\eta$ as the temperature increases. Let us focus on the MD data. In Figure 4 we see that the a-SiO ${ }_{2} \mathrm{OH}$ enhancement slightly decreases in passing from 450 to $550 \mathrm{~K}$. Even if, in absolute values, $\mathrm{c}_{P, n m}(550 \mathrm{~K})>\mathrm{c}_{P, n m}(450 \mathrm{~K})$ (see Table 2$)$, the $\eta$ decrease is due to the fact that the $\mathrm{c}_{P, s m}$ of $\mathrm{KNO}_{3}$ slightly increases with $T$. In the a-C case instead, there is a $\approx 5 \%$ increase in $\eta$ between the same temperatures, with the same $\mathrm{c}_{P, s m}$ increase. Looking at Figure 5, this increase happens at similar (or even reduced with $T$ ) interface region volume. Thus, the larger $c_{p}$ enhancement with $T$ in a-C must come from an increased vibration of the NPs as a whole due to temperature. The effect is not observed in a- $\mathrm{SiO}_{2} \mathrm{OH}$, where this vibrational motion is small at both temperatures, due to the absence of an excluded volume region.

The large a-C $c_{p}$ enhancements, reaching a substantial value of $110 \%$ (for $\sigma_{n p}=2$ $\mathrm{nm}, T=550 \mathrm{~K}$ and $x_{n p}=0.1$ (see Figure 7 ) is then the result of a set of non-trivial properties, which was possible to rationalize only via the combined use of our theoretical and computational analyses.

Nano-holes in solid $\mathbf{K N O}_{3}$. The two previous systems highlighted the crucial role of different NP-SM cross interactions in building the interface region properties. It is then interesting to consider the limiting case where the NP is replaced by an empty space (nano-hole). Here, neither repulsion nor attraction are, obviously, present and no NP vibrational motion can be associated to the nano-holes. Still, we can expect a relaxation of the $\mathrm{KNO}_{3}$ atoms into the hole, counterbalanced by the cohesive energy of the solid. MD calculations in similar conditions as in previous systems (a nano-hole with $\sigma=5.4$ $\mathrm{nm}$ in $\mathrm{KNO}_{3}$ at 450 and $550 \mathrm{~K}$ ) have been performed. ${ }^{7}$ Density profiles around the nanoholes are shown in Figure 8. Looking at the edge of the nano-hole (vertical dotted line)

\footnotetext{
${ }^{7}$ Note that it is not possible, with nano-holes, to make extrapolations as a function of $x_{n p}$ or $\sigma$, as in this case $x_{n p}=0$.
} 


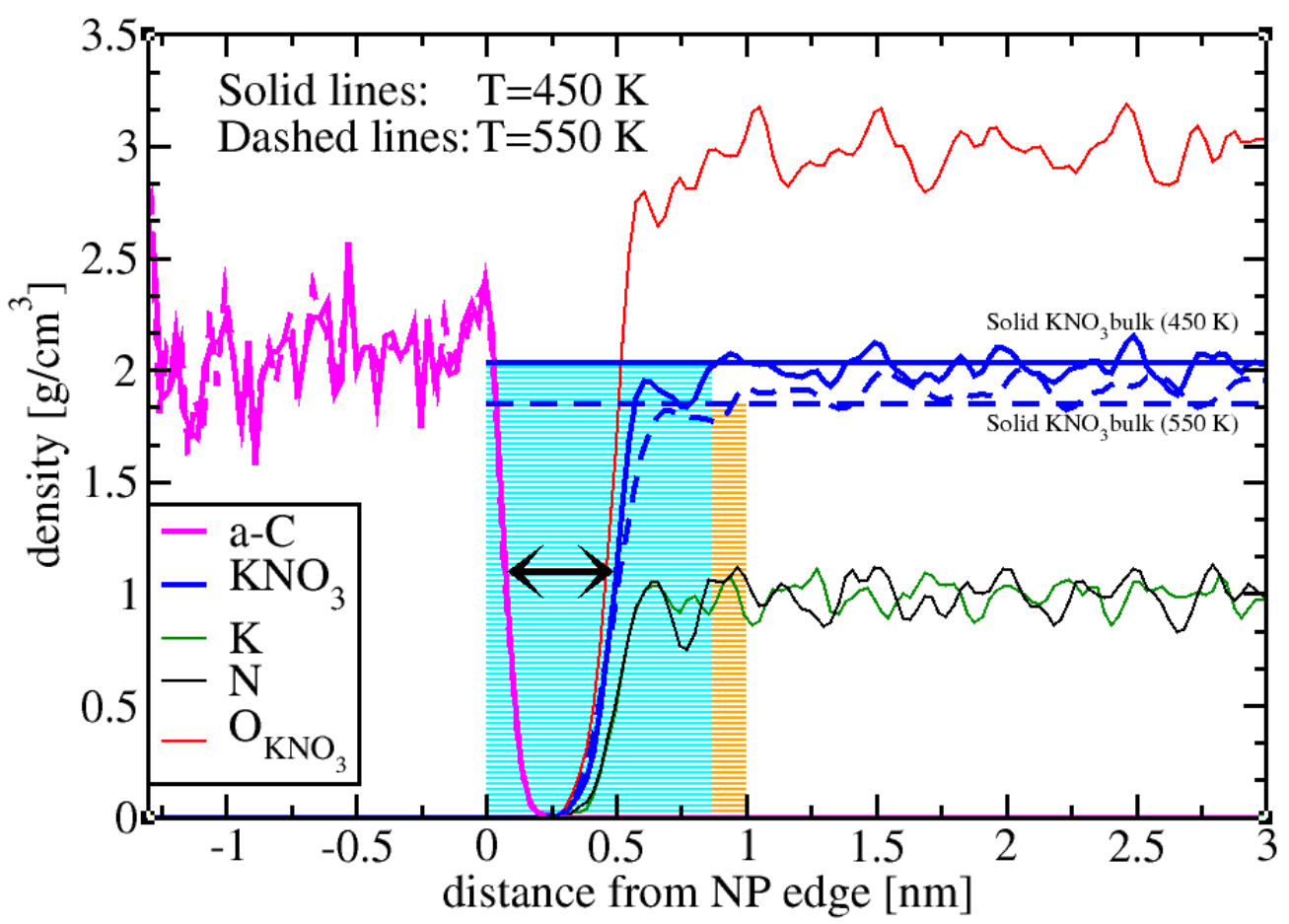

Figure 6: Density profiles of solid $\mathrm{KNO}_{3}$ around an amorphous carbon (a-C) NP at $T=450$ and $550 \mathrm{~K}$. Plots and highlighted regions have the same meaning as in Figure 3. Black arrows indicate the excluded volume region around the NP, as described in the text.

we see that the cohesive energy of the solid dominates the shape of the $\mathrm{KNO}_{3}$ relaxed density profile, which exhibits a depletion between 0 and $\approx 1 \mathrm{~nm}$.

Table 2 shows a finite excess term at both temperatures, due to "interfacial" atomic re-arrangements, similar to the ones in previous systems. This confirms the mechanism of interfacial processes which causes $c_{p}$ variations, no matter what the NP material is, and even in the absence of it. The excess terms are lower than in a- $\mathrm{SiO}_{2} \mathrm{OH}$ and a-C in solid $\mathrm{KNO}_{3}$, even if, as shown in Figure 8, the extension of the "interface" region lies in between those of a-SiO ${ }_{2} \mathrm{OH}$ and a-C (Figures 3 and 6). This apparently counter-intuitive result confirms that in the a-C case, a large contribution to the excess term is given by the NPs vibrational motion.

Finally, the nano-hole concept serves to understand the various mechanisms of $\mathrm{c}_{p}$ enhancement, but is also very relevant in applications as solid nanomaterials where pores or interconnected cavities are present (e.g. foams). ${ }^{6,7,14,42}$ 


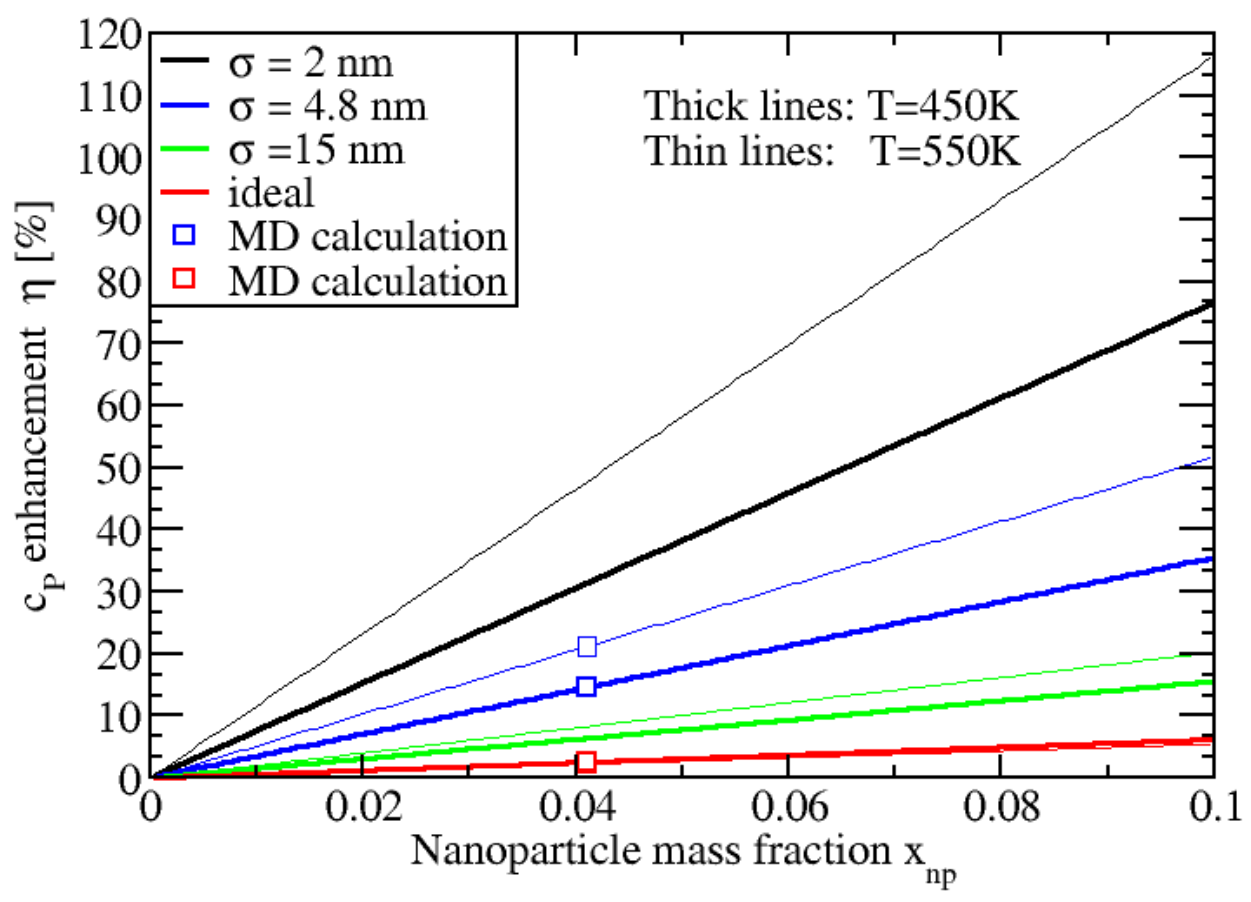

Figure 7: Specific heat enhancement as in Figure 4, but for amorphous $\mathrm{C}$ (a-C) nanoparticles in solid $\mathrm{KNO}_{3}$. Note the $\approx 110 \%$ enhancement for $\sigma=2 \mathrm{~nm}$ at $T=550 \mathrm{~K}$ and $x_{n p}=0.1$. Unlike in the a- $\mathrm{SiO}_{2} \mathrm{OH}$ case, no plot is truncated, as here large values of mass fraction are still compatible with a dilute regime for all NP sizes (see SI for details and Table SI-8), being the interface region rather small. 


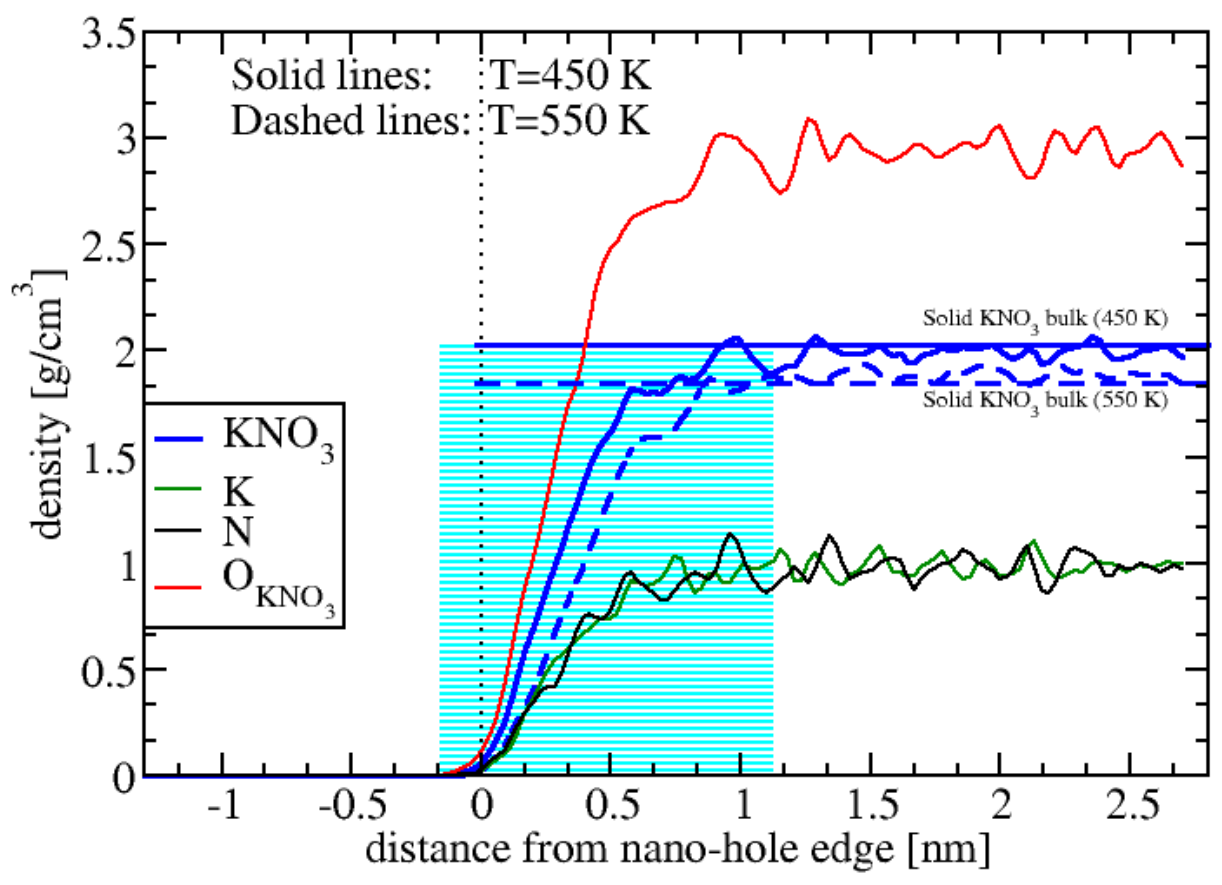

Figure 8: Density profiles of solid $\mathrm{KNO}_{3}$ around a nano-hole at $T=450$ and $550 \mathrm{~K}$. Plots and highlighted regions have the same meaning as in Figure 3. The initial edge of the nano-hole is indicated as a dotted vertical line. 


\section{Specific heat of liquid nanomaterials}

We now turn to nanomaterials made of NPs in a liquid suspending medium. We will analyze systems analogue to the ones in the previous Section, but in presence of liquid $\mathrm{KNO}_{3}$. These systems give, in general, qualitatively and quantitatively different results as compared to the corresponding solid systems.

a-SiO ${ }_{2} \mathrm{OH}$ nanoparticles in liquid $\mathbf{K N O}_{3}$. First we consider a- $\mathrm{SiO}_{2} \mathrm{OH}$ spherical NPs in liquid $\mathrm{KNO}_{3}$. We performed $\mathrm{MD}$ simulations at 650 and $800 \mathrm{~K}$, at ambient pressure $(P=1 \mathrm{~atm})$. These temperatures have been chosen in a range far away from the melting point $\left(\mathrm{T}_{M}=607 \mathrm{~K}\right)$ and still in the regime of liquid stability. ${ }^{8}$

Table 3: Calculated $\mathrm{c}_{p}$ for liquid nanomaterials, using $\mathrm{KNO}_{3}$ as suspending medium. NPs have diameter $\sigma=4.8-5.2 \mathrm{~nm}$. Temperatures $(\mathrm{T})$ are given in $\mathrm{K}, \mathrm{c}_{p}$ 's in $\mathrm{JK}^{-1} \mathrm{~g}^{-1}$ and $C_{A, e x}$ in $10^{-7} \times \mathrm{JK}^{-1} \mathrm{~g}^{-1} \mathrm{~cm}^{-2}$.

\begin{tabular}{|c|c|c|c|c|c|c|c|c|}
\hline & $\mathrm{T}$ & $c_{P, n p}$ & $c_{P, s m}$ & $x_{n p}$ & $c_{P, n m}^{i d}$ & $c_{P, n m}$ & $c_{P, e x}$ & $C_{A, e x}$ \\
\hline \multirow{2}{*}{$\mathrm{a}_{-} \mathrm{SiO}_{2} \mathrm{OH}$} & 650 & 1.311 & 1.518 & 0.08198 & 1.501 & 1.457 & -0.044 & -1.06 \\
\cline { 2 - 9 } $\mathrm{NP}$ & 800 & 1.506 & 1.518 & 0.08198 & 1.517 & 1.483 & -0.034 & -0.82 \\
\hline $\mathrm{a}-\mathrm{C}$ & 650 & 2.130 & 1.518 & 0.0542 & 1.551 & 1.618 & 0.130 & 3.32 \\
\cline { 2 - 9 } $\mathrm{NP}$ & 800 & 2.153 & 1.518 & 0.0542 & 1.552 & 1.618 & 0.129 & 3.29 \\
\hline
\end{tabular}

The $c_{p}$ results are shown in Table 3. The $\mathrm{c}_{P, n m}$ values are 1.457 and $1.483 \mathrm{JK}^{-1} \mathrm{~g}^{-1}$ at $650 \mathrm{~K}$ and $800 \mathrm{~K}$, respectively, higher than in the NM solid regime. Importantly though, we find negative excess terms $c_{P, e x}$, indicating that the interface region in the liquid NM plays an opposite role as compared to the solid. At the same temperatures, $\mathrm{c}_{P, s m}=$ 1.518 $\mathrm{JK}^{-1} \mathrm{~g}^{-1},{ }^{9}$ meaning that in the liquid NM there is a "negative enhancement" with respect to the suspending medium, as shown in Figure 9. As in the solid system, $\mathrm{c}_{P, n p}$ is lower than $\mathrm{c}_{P, s m}$, thus $\mathrm{c}_{P, n m}^{i d}$ decreases and the enhancement becomes even more negative for larger $x_{n p}$, as it can be seen by the negative slopes of the linear plots in Figure 9. The stronger decrease is obtained for the smallest NP, while an ideal system decrease is approached by increasing the NP diameter.

Let us give a rationale of the negative excess terms $c_{P, e x}$. Eq. (4) indicates that the specific heat of the interface region $\left(c_{P, \text { int }}\right)$ must be smaller than in the $\mathrm{KNO}_{3}$ liquid bulk

\footnotetext{
${ }^{8}$ Experimentally, $\mathrm{KNO}_{3}$ starts to decompose in $\mathrm{KNO}_{2}$ and $\mathrm{O}_{2}$ already at $673 \mathrm{~K}$, but still the system remains liquid

${ }^{9} \mathrm{KNO}_{3}$ specific heat is temperature independent in the liquid regime. ${ }^{22}$
} 


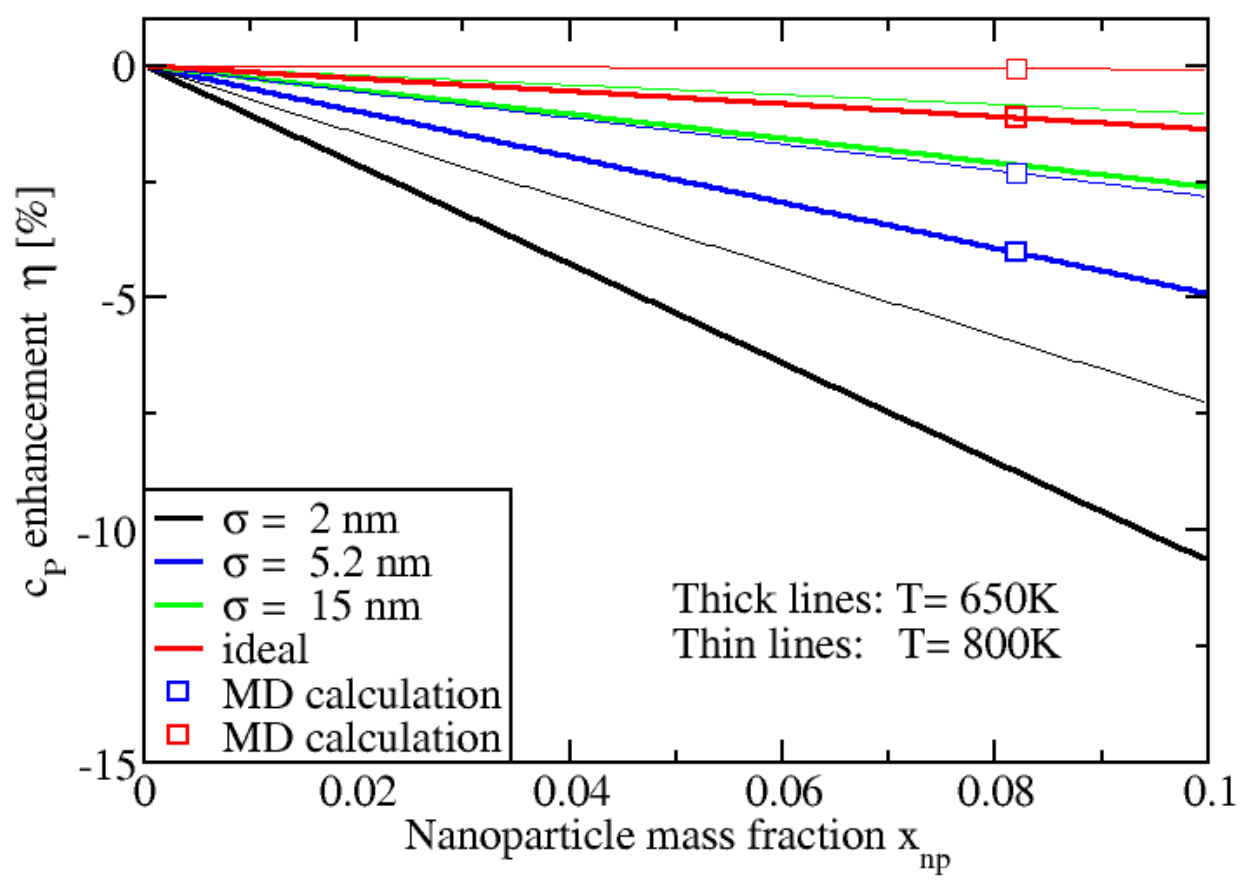

Figure 9: Specific heat enhancement as in Figure 4, but for a- $\mathrm{SiO}_{2} \mathrm{OH}$ nanoparticles in liquid $\mathrm{KNO}_{3}$, at $T=650$ and $800 \mathrm{~K}$.

$\left(c_{P, s m}\right)$, toward values typical of a solid phase. In Figure 10, we show the density profiles of the interface region at 650 and $800 \mathrm{~K}$. The interface region in liquids extends till the SM density does not exhibit any oscillations. As anticipated, beyond the interpenetrating region the $\mathrm{KNO}_{3}$ density exhibits strong oscillations (between $\approx 0.4$ and $1.5 \mathrm{~nm}$ ) and "piles up" at the interface. The density reaches values higher than the liquid bulk at both temperatures, indicating a NP-SM attraction which prevails over the cohesive energy of the liquid. The pile-up is absent in the solid (Figure 3), which instead exhibits a depletion. Starting from the NP surface, Figure 10 shows an alternation of shells made of $\mathrm{K}^{+}$and $\mathrm{NO}_{3}{ }^{-}$ions, with the presence of peaks and dips in counter-phase (dark green and black/red plots). The shell structure vanishes at $\approx 2 \mathrm{~nm}$ from the NP surface and, importantly, it remains stable with the increasing temperature (see also below).

Thus, in a somehow complementary picture as compared to the solid NM, $(i)$ the decreased $c_{P, \text { int }}$ of the interface, $(i i)$ its pronounced shell structure, $(i i i)$ its stability with temperature and $(i v)$ its increased density, indicate that the interface behaves as a "stable 
solid-like" region, with respect to the liquid bulk. The presence of a solid-like layer has been already suggested in the literature, to explain experimental data. ${ }^{11-13,20,43}$ However, its presence was introduced to justify a $c_{p}$ increase, rather than a decrease. ${ }^{20,43-45}$ The rationale was the hypothesis of a melting solid-like region, partially incorporating the heat of fusion. ${ }^{43}$ However, a melting process is clearly ruled out by our calculations, showing that the shell structure is not destroyed with temperature.

Hence, the interface region must be characterized by harder vibrational modes, less prone to absorb energy than the liquid modes. ${ }^{46-49}$ The replacement of softer liquid modes with harder "solid like" modes is the leading (negative) contribution to $c_{P, e x}{ }^{10}$

Finally, the temperature independence of the shell region structure (there is only a rigid downshift of the density profile from 650 to $800 \mathrm{~K}$ ) as well as of the $c_{P, s m}$, implies that also $c_{P, e x}$ is only slightly dependent on $T$ (see Table 3 ).

Carbon nanoparticles in liquid $\mathbf{K N O}_{3}$. Amorphous carbon NPs in liquid $\mathrm{KNO}_{3}$ inherit several properties already present in the corresponding solid system. Moreover, the nanomaterial has some missing features as compared to the a- $\mathrm{SiO}_{2} \mathrm{OH}$ NPs in liquid $\mathrm{KNO}_{3}$. As shown in Figure 11, the excluded volume region observed in the solid is also obtained in the liquid. Unlike in the liquid containing a- $\mathrm{SiO}_{2} \mathrm{OH} \mathrm{NPs}$, there is no density "pile up" near the a-C NP, due to the purely repulsive NP-SM cross terms. The $\mathrm{KNO}_{3}$ density oscillations are also strongly reduced (compare, for example, the first peak at $0.6 \mathrm{~nm}$ of $\approx 1.1$ height of the $\mathrm{K}$ profiles in Figure 11 with the one at $0.4 \mathrm{~nm}$ of $\approx 1.7$ height in Figure 10) and, in fact, there is no shell structure with ions densities in counter-phase. These facts indicate, essentially, the absence of a perturbed structure surrounding the carbon NP. Thus, negative contributions to the excess terms due to a more "solid-like" behaviour of the liquid are ruled out.

As in the solid, we obtain positive values of the excess terms, as illustrated in Table 3. The $c_{p}$ enhancement $\eta$ is presented in Figure 12, showing that enhancement values arrive to $\approx 25 \%$ for $\sigma=2 \mathrm{~nm}$ and $x_{n p}=0.1$. Such enhancements in a liquid system

\footnotetext{
${ }^{10}$ An extended shell structure augments, effectively, the displaced volume during the NP motion. We then expect also the presence of hydrodynamic waves in the SM. These happen at larger time-scales than our MD simulation time, thus their possible effects on the $c_{p}$ are not included in our calculations.
} 


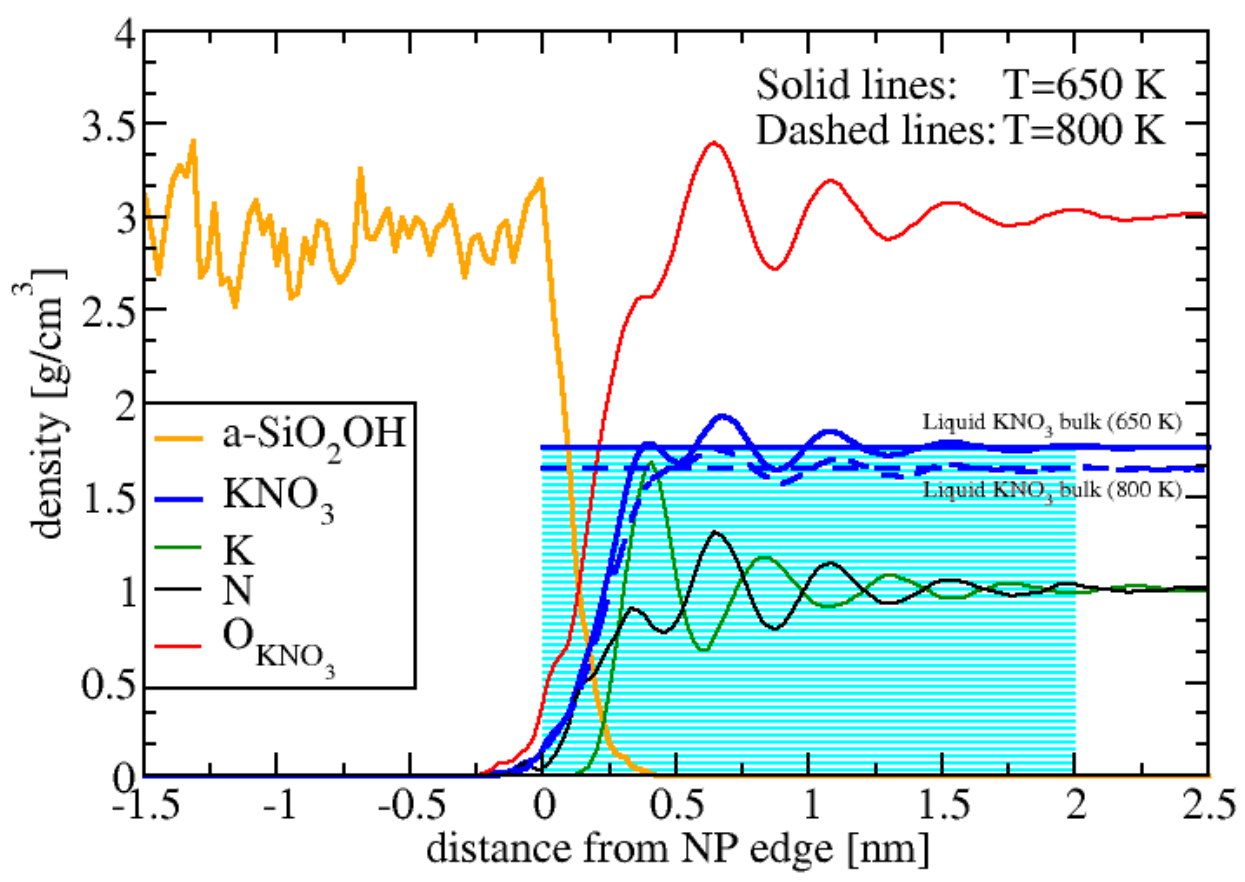

Figure 10: Density profiles of liquid $\mathrm{KNO}_{3}$ around an a- $\mathrm{SiO}_{2} \mathrm{OH}$ nanoparticle at $T=650$ and $800 \mathrm{~K}$. Plots and highlighted regions have the same meaning as in Figure 3. Note the counter-phase behaviour of the $\mathrm{K}^{+}$and $\mathrm{NO}_{3}{ }^{-}$density profiles between $\approx 0.35$ and 2 $\mathrm{nm}$, indicating a shell structure. 
are extremely relevant for industrial applications in CSP plants, which routinely operate with liquid TES materials, with molten salts as suspending media.

Due to the similarity of the density profiles with temperature, and the fact that both $\mathrm{c}_{P, s m}$ and $\mathrm{c}_{P, n m}^{i d}$ are temperature independent, the resulting excess terms do not change at 650 and $800 \mathrm{~K}$.

The contributing factors to these large and positive enhancements are the same as in the solid case: $(i)$ a $\mathrm{c}_{P, n p}$ higher than $\mathrm{c}_{P, s m} ;(i i)$ an excluded volume region allowing vibrations of the whole nanoparticle; ${ }^{46}$ (iii) the fact that the a-C nanoparticles are light; (iv) the absence of a interface region with "solid-like" behaviour, that would reduce the enhancement.

Finally, we can validate the predictive capabilities of our theoretical model in obtaining the $c_{p}$ of systems with a large intervals of NPs sizes and mass fractions. In Figure 12 we show a plot (dashed orange line) of the enhancement $\eta$ for NPs with $\sigma=4 \mathrm{~nm}$, obtained via Eq. (8) of the model after a MD calculation for NPs having $\sigma=5 \mathrm{~nm}$ and $x_{n p} \approx 0.054$ (blue square point). Then, we performed a separate MD calculation of $\eta$ at $\sigma=4 \mathrm{~nm}$ and $x_{n p} \approx 0.033$ (orange/filled black point). We obtain an excellent agreement between MD and model (the MD point is on top of the dashed orange line). This represents a strong validation of the model, which allows, at no computational cost, to obtain $\mathrm{c}_{p}$ enhancements in a large range of NPs sizes and mass fractions, after a single MD calculation at specific $x_{n p}$ and $\sigma$ values (and at a given temperature). 


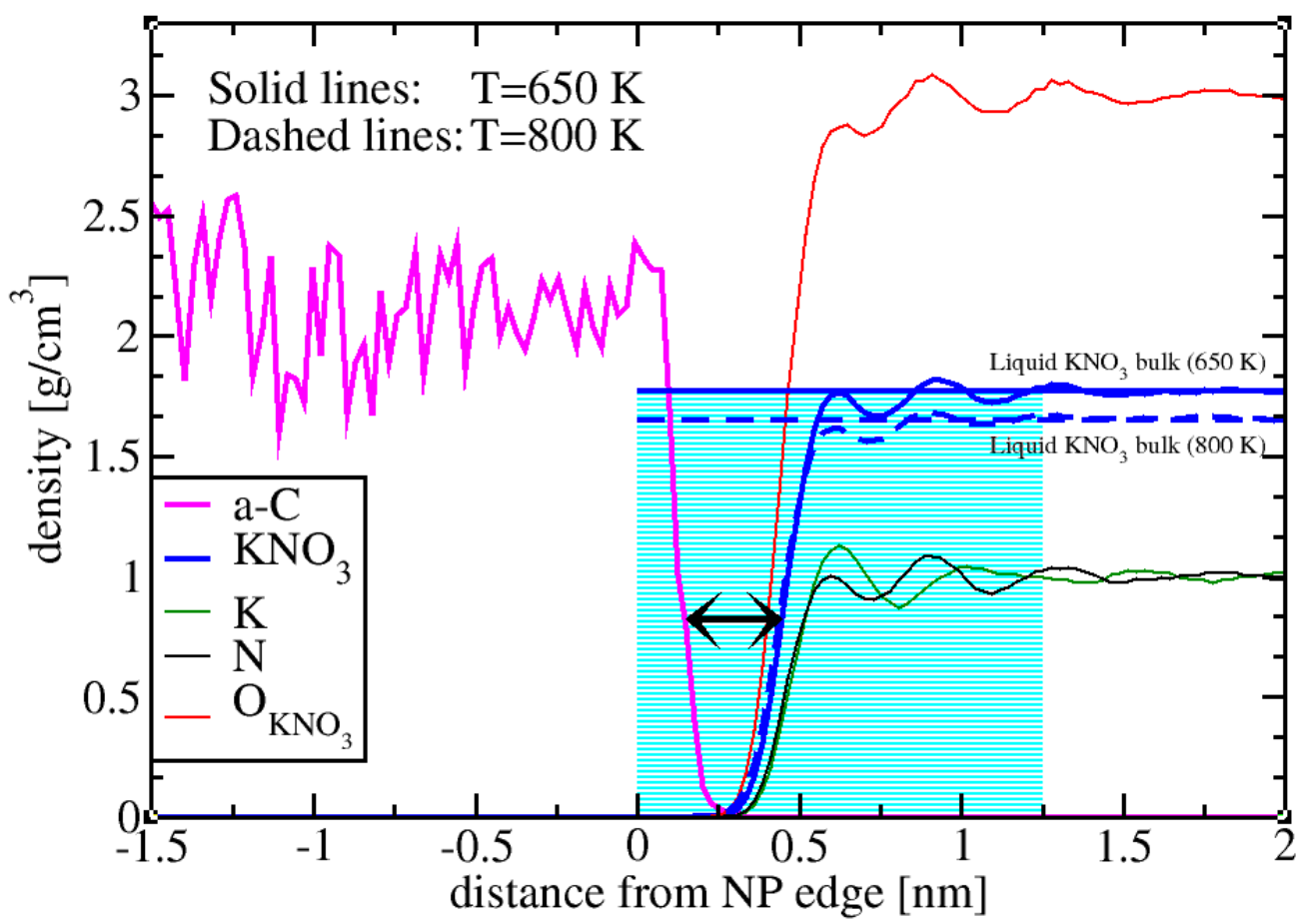

Figure 11: Density profiles as in Figure 3, but for liquid $\mathrm{KNO}_{3}$ around a carbon NP, at $T=650$ and $800 \mathrm{~K}$. As in the solid case, an excluded volume region around the NP exists, indicated by black arrows.

\section{Summary for solid and liquid nanomaterials}

We summarize here our results obtained with $\mathrm{KNO}_{3}$ as suspending medium.

For solid nanomaterials we find:

- Positive $c_{P, e x}$ and $C_{A, e x}$ excess terms in all analyzed NMs (for attractive/repulsive and purely repulsive cross interaction potentials), implying positive $\mathrm{c}_{p}$ enhancements with respect to the ideal specific heat, $c_{P, n m}^{i d}$.

- Positive enhancements with respect to the specific heat of the suspending medium, $\mathrm{c}_{P, s m}$, when the a-SiO $2 \mathrm{OH}$ NPs diameter is less than $15 \mathrm{~nm}$, and for all a-C NP diameters.

For liquid nanomaterials, we find:

- Positive $c_{P, e x}$ and $C_{A, e x}$ excess terms for all a-C analyzed NMs. The $c_{p}$ increases both with respect to the ideal $\mathrm{c}_{P, n m}^{i d}$ and the $\mathrm{c}_{P, s m}$ of the suspending medium.

- In $\mathrm{NMs}$ containing $\mathrm{a}-\mathrm{SiO}_{2} \mathrm{OH}$ nanoparticles, $\mathrm{c}_{p}$ decreases both with respect to $\mathrm{c}_{P, n m}^{i d}$ and $\mathrm{c}_{P, s m}$, in all considered (dilute regime) cases. 


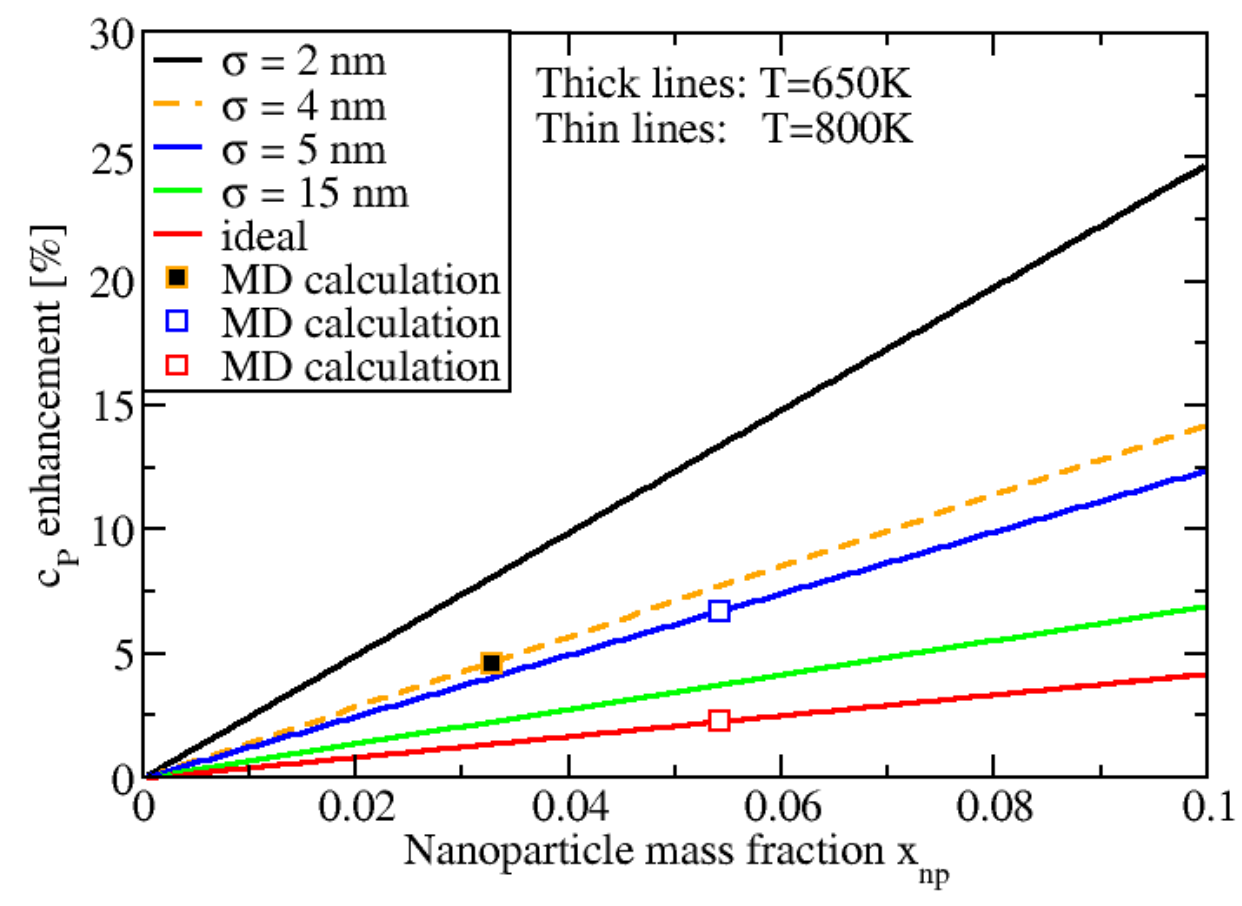

Figure 12: Specific heat enhancement as in Figure 7, but for amorphous $\mathrm{C}$ nanoparticles in liquid $\mathrm{KNO}_{3}$, at $T=650$ and $800 \mathrm{~K}$. The dashed orange line is obtained via the theoretical model (Eq. (8)) for NPs with $\sigma=4 \mathrm{~nm}$, obtained after a MD calculation at $\sigma=5 \mathrm{~nm}$ and $x_{n p} \approx 0.054$ (blue square point). A separate MD calculation at $\sigma=4 \mathrm{~nm}$ and $x_{n p} \approx 0.033$ is shown with an orange/filled black point, in excellent agreement with the model. 
We find the following main contributions to the $\mathrm{c}_{p}$ variations:

1. An atomic re-organization at the interface, strongly material and phase dependent. In interface regions, we found a more "liquid-like" ("solid-like") behaviour in a solid (liquid) bulk suspending medium, with consequences on the vibrational modes and $c_{p}$, in line with other studies. ${ }^{46-49}$

2. The possibility to have vibrational modes of the NP as a whole, when the NP-SM interaction creates an excluded volume region around the NP.

3. The relation between the $c_{P, n p}$ of the NPs and the one of the suspending medium, $\mathrm{c}_{P, s m}$.

The three contributions are often competing and may lead to opposite contributions to the $c_{p}$ variation. Note that in all analyzed nanomaterials the NPs mass fractions are always well inside the dilute regime (see SI). Thus, any agglomeration/aggregation effect on $c_{p}$ is excluded from our analysis. In particular, the negative enhancements in liquid $\mathrm{KNO}_{3}$ containing a-SiO $2 \mathrm{OH}$ NPs are not related to these effects.

\section{Conclusions}

In this work, we combined a theoretical model with extensive molecular dynamics (MD) simulations to analyze, in a unified description, the variation mechanisms of the specific heat in ionic bulk materials containing nanoparticles (NMs). We consider technologically relevant NMs, made by the inclusion of nanoparticles in solid and liquid suspending media.

We considered three classes of NMs: systems where NP-SM effective interactions are attractive and repulsive (represented by silica NPs in $\mathrm{KNO}_{3}$ ); systems where these interactions are purely repulsive (amorphous carbon NPs in $\mathrm{KNO}_{3}$ ); systems with empty cavities (nano-holes in $\mathrm{KNO}_{3}$ ).

Our theoretical model, for each NM and phase and on the basis of MD calculations at a given temperature, NP diameter $(\sigma)$ and NP mass fraction $\left(x_{p}\right)$, allows extrapolations 
at different $\sigma$ and $x_{p}$, at no extra computational cost. Our theory and simulations allow to analyse $c_{p}$ trends in a very large space of "parameters", such as the involved materials, their phase, the NPs size and concentration, the temperature. We also introduce a purely material dependent parameter $C_{A, e x}$ (excess term per NP unit surface), crucial to determine the sign and the entity of the $c_{p}$ variations.

As compared to pure $\mathrm{KNO}_{3}$, in solid NMs we find enhancements up to $110 \%$ by adding carbon NPs at $550 \mathrm{~K}$, when $x_{p}=0.1$ and $\sigma=2 \mathrm{~nm}$. In liquids, at 650-800 K, enhancements with the same NPs and mass fractions arrive to $\approx 25 \%$. More modest $\mathrm{c}_{p}$ enhancements (up to $\approx 10 \%$ ), and even diminutions, are obtained in solid and liquid $\mathrm{KNO}_{3}$ containing a-SiO${ }_{2} \mathrm{OH}$ NPs, respectively.

Our analysis is based on systems that represent a large set of nanomaterials. Suspending media similar to $\mathrm{KNO}_{3}$, relevant in industrial applications, are expected to give similar trends (in CSP plants based on parabolic troughs and towers, the storage tanks are filled with liquid nitrates). Thus, tentative criteria for large $c_{p}$ enhancements at a given mass fraction can be established. Increments are expected when the two materials have a large $C_{A, e x}(T)$ term; when the nanoparticles are light; when their size is small; when their specific heat $\mathrm{c}_{P, n p}$ is larger than the one of the hosting medium $\mathrm{c}_{P, s m}$; in solid, rather than liquid suspending media. In liquids, the sign of $\mathrm{c}_{p}$ variations strongly depends on the nature of interactions at the interface.

We believe that in this unified, non-trivial scenario of competing effects, our results for liquids systems help in the interpretation of controversial experimental trends reported in the literature, ${ }^{10-14,45}$ where, to date, there are no direct measurements of the SM structuring/restructuring around the NP surface. While for the solids, we identify a systematic, and reproducible way of improve the thermal properties using solid, ionic bulk nanomaterials. 


\section{Methods}

\section{Classical Molecular Dynamics (MD)}

MD simulations have been performed using the LAMMPS software package. ${ }^{50,51}$ For all considered systems, the $\mathrm{c}_{p}$ values of have been calculated from the enthalpy data obtained in the NPT statistical ensemble.

For simulations of an a- $\mathrm{SiO}_{2} \mathrm{OH} \mathrm{NP}$ in liquid and solid $\mathrm{KNO}_{3}$ we used a time-step of 1 fs. Systems have been equilibrated using $10^{6}$ time-steps and run with further $5 \times 10^{5}$ $-5 \times 10^{6}$ time-steps. For the solid (liquid) systems, equilibrated configurations at the highest (lowest) temperature have been used as input for the closest next lower (higher) temperature.

Other relevant parameters: Nose-Hoover barostat time constant of 0.5 ps; a NoseHoover thermostat time constant of 0.1 ps. A Buckingham and Coulomb interaction cutoff distance of $r_{c}=1.2 \mathrm{~nm}$ for systems with $\mathrm{K}^{+}$ions, and $r_{c}=1.1 \mathrm{~nm}$ for other cases; long-range calculation accuracy for forces is $10^{-4}$.

\section{Acknowledgement}

Via our membership of the UK's HEC Materials Chemistry Consortium, which is funded by EPSRC (EP/L000202, EP/R029431), this work used the ARCHER UK National Supercomputing Service (http://www.archer.ac.uk). This work has been carried out with the financial support of the "Regione Autonoma della Sardegna". BD acknowledges VIT University and the Centre for Nanotechnology Research for providing extra resources to carry out the work.

\section{Supporting Information Available}

Simulated systems, Definition of dilute regime, Nanoparticles parameters, MD interaction potentials, Cross interaction potential terms between NPs and SM, Derivation of the $x_{s m, i n t}$ expression (Eq. 6), Derivation of the $c_{P, e x}(T, \sigma)$ excess term (Eqs. 7 and 8), 
Modification of NPs surface due to the interaction with the suspending medium. This material is available free of charge via the Internet at http://pubs.acs.org.

\section{References}

1. Santamarta, J. Concentrated Solar Power Had a Global Total Installed Capacity of 6,451 MW in 2019. 2019; http://www.evwind.es/2020/02/02/ concentrated-solar-power-had-a-global-total-installed-capacity-of-6451-mw-in-2019 73360, Available on line (accessed Dec 2, 2020).

2. SunShot 2030. 2016; https://www.energy.gov/eere/solar/sunshot-2030, Available on line (accessed Dec 2, 2020).

3. Glatzmaier, G. Summary Report for Concentrating Solar Power Thermal Storage Workshop: New Concepts and Materials for Thermal Energy Storage and HeatTransfer Fluids, May 20, 2011. 2011,

4. Kim, W.; Wang, R.; Majumdar, A. Nanostructuring Expands Thermal Limits. Nano Today 2007, 2, $40-47$.

5. Zhou, Z.; Zhang, H.; Zhou, Y.; Qiao, H.; Gurung, A.; Naderi, R.; Elbohy, H.; Smirnova, A. L.; Lu, H.; Chen, S.; Qiao, Q. Binder Free Hierarchical Meso-Porous Carbon Foam for High Performance Lithium Ion Battery. Scientific Reports 2017, $7,1440$.

6. Karthik, M.; Faik, A.; Doppiu, S.; Roddatis, V.; D'Aguanno, B. A Simple Approach for Fabrication of Interconnected Graphitized Macroporous Carbon Foam with Uniform Mesopore Walls by Using Hydrothermal Method. Carbon 2015, 87, $434-443$.

7. Karthik, M.; Faik, A.; D’Aguanno, B. Graphite Foam as Interpenetrating Matrices for Phase Change Paraffin Wax: A Candidate Composite for Low Temperature Thermal Energy Storage. Solar Energy Materials and Solar Cells 2017, 172, 324 - 334. 
8. Ubbelohde, A.; Lewis, F.; Rice, S. A. Graphite and Its Crystal Compounds. Physics Today 1961, 14, 66.

9. Gao, J.; Ndong, R. S.; Shiflett, M. B.; Wagner, N. J. Creating Nanoparticle Stability in Ionic Liquid [C4mim][BF4] by Inducing Solvation Layering. ACS Nano 2015, 9, $3243-3253$.

10. Arthur, O.; Karim, M. An Investigation into the Thermophysical and Rheological Properties of Nanofluids for Solar Thermal Applications. Renewable and Sustainable Energy Reviews 2016, 55, 739 - 755 .

11. Riazi, H.; Mesgari, S.; Ahmed, N. A.; Taylor, R. A. The Effect of Nanoparticle Morphology on the Specific Heat of Nanosalts. International Journal of Heat and Mass Transfer 2016, 94, $254-261$.

12. Riazi, H.; Murphy, T.; Webber, G. B.; Atkin, R.; Tehrani, S. S. M.; Taylor, R. A. Specific Heat Control of Nanofluids: A Critical Review. International Journal of Thermal Sciences 2016, 107, 25 - 38.

13. Hentschke, R. On the Specific Heat Capacity Enhancement in Nanofluids. Nanoscale Research Letters 2016, 11, 88.

14. Liu, M.; Fernández, A. I.; Segarra, M. In High Temperature Thermal Storage Systems Using Phase Change Materials; Cabeza, L. F., Tay, N. H. S., Eds.; Academic Press (Elsevier), London, 2018; pp $195-230$.

15. Xie, Q.; Zhu, Q.; Li, Y. Thermal Storage Properties of Molten Nitrate Salt-Based Nanofluids with Graphene Nanoplatelets. Nanoscale Research Letters 2016, 11, 306.

16. International Energy Agency (IAE), Technology Roadmap, Energy storage. 2014.

17. Shin, D.; Banerjee, D. Enhancement of Specific Heat Capacity of High-Temperature Silica-Nanofluids Synthesized in Alkali Chloride Salt Eutectics for Solar ThermalEnergy Storage Applications. International Journal of Heat and Mass Transfer 2011, $54,1064-1070$. 
18. Benmansour, A.; Hamdan, M.; Bengeuddach, A. Experimental and Numerical Investigation of Solid Particles Thermal Energy Storage Unit. Applied Thermal Engineering 2006, 26, $513-518$.

19. Schuller, M.; Shao, Q.; Lalk, T. Experimental Investigation of the Specific Heat of a Nitrate-Alumina Nanofluid for Solar Thermal Energy Storage Systems. International Journal of Thermal Sciences 2015, 91, 142 - 145.

20. Shin, D.; Banerjee, D. Enhanced Specific Heat Capacity of Nanomaterials Synthesized by Dispersing Silica Nanoparticles in Eutectic Mixtures. Journal of Heat Transfer 2013, 135, 032801-032808.

21. Engelmann, S.; Hentschke, R. Specific Heat Capacity Enhancement Studied in Silica Doped Potassium Nitrate via Molecular Dynamics Simulation. Scientific Reports 2019, 9, 7606 .

22. D’Aguanno, B.; Karthik, M.; Grace, N.; Floris, A. Thermostatic Properties of Nitrate Molten Salts and Their Solar and Eutectic Mixtures. Scientific Reports 2018, 8, 10485.

23. Adya, A. K.; Takagi, R.; Kawamura, K.; Mikami, M. Structural Determination of Molten NaNO3, NaNO2 and Their Eutectic Mixture by Molecular Dynamics Simulation and X-Ray Diffraction. Molecular Physics 1987, 62, 227-238.

24. Bródka, A.; Zerda, T. W. Properties of Liquid Acetone in Silica Pores: Molecular Dynamics Simulation. The Journal of Chemical Physics 1996, 104, 6319.

25. Spohr, E.; Hartnig, C.; Gallo, P.; Rovere, M. Water in Porous Glasses. A Computer Simulation Study. Journal of Molecular Liquids 1999, 80, 165 - 178.

26. Kissa, M. Dispersions: Characterizations Testing, and Measurement; Marcel Dekker, New York, 1999.

27. Metin, C. O.; Lake, L. W.; R., M. C.; Nguyen, Q. P. Stability of Aqueous Silica Nanoparticle Dispersions. J. Nanopart. Res. 2011, 13, 839850. 
28. Atkins, P. Physical Chemistry; Oxford University Press, Oxford, 2018.

29. Sandler, S. I. Chemical, Biochemical, And Engineering Thermodynamics; Wiley, New York, 2017.

30. Ackland, G. J.; Jones, A. P. Applications of Local Crystal Structure Measures in Experiment and Simulation. Physical Review B 2006, 73, 054104.

31. Bose, S. Molecular Dynamics Modelling of Thermostatic Properties of Nanoparticles. M.Sc. thesis, Vellore Institute of Technology, 2020.

32. Lu, M.-C.; Huang, C.-H. Specific Heat Capacity of Molten Salt-Based Alumina Nanofluid. Nanoscale Research Letters 2013, 8, 292.

33. Naicker, P. K.; Cummings, P. T.; Zhang, H.; Banfield, J. F. Characterization of Titanium Dioxide Nanoparticles Using Molecular Dynamics Simulations. The Journal of Physical Chemistry B 2005, 109, 15243-15249.

34. Zhuravlev, L. The Surface Chemistry of Amorphous Silica. Zhuravlev Model. Colloids and Surfaces A: Physicochemical and Engineering Aspects 2000, 173, 1 - 38.

35. Takahashi, Y.; Sakamoto, R.; Kamimoto, M. Heat Capacities and Latent Heats of LiNO3, NaNO3, and KNO3. International Journal of Thermophysics 1988, 9, 10811090.

36. Wang, B.-X.; Zhou, L.-P.; Peng, X.-F. Surface and Size Effects on the Specific Heat Capacity of Nanoparticles. International Journal of Thermophysics 2006, 27, 139151.

37. Saeedian, M.; Mahjour-Shafiei, M.; Shojaee, E.; Mohammadizadeh, M. R. Specific Heat Capacity of TiO2 Nanoparticles. Journal of Computational and Theoretical Nanoscience 2012, 9, 616-620.

38. Chieruzzi, M.; Miliozzi, A.; Crescenzi, T.; Torre, L.; Kenny, J. M. A New Phase Change Material Based on Potassium Nitrate with Silica and Alumina Nanoparticles for Thermal Energy Storage. Nanoscale Research Letters 2015, 10, 273. 
39. Chieruzzi, M.; Cerritelli, G. F.; Miliozzi, A.; Kenny, J. M.; Torre, L. Heat Capacity of Nanofluids for Solar Energy Storage Produced by Dispersing Oxide Nanoparticles in Nitrate Salt Mixture Directly at High Temperature. Solar Energy Materials and Solar Cells 2017, 167, $60-69$.

40. Dudda, B.; Shin, D. Effect of Nanoparticle Dispersion on Specific Heat Capacity of a Binary Nitrate Salt Eutectic for Concentrated Solar Power Applications. International Journal of Thermal Sciences 2013, 69, 37 - 42.

41. Dudda, B.; Shin, D. Investigation of Molten Salt Nanomaterial as Thermal Energy Storage in Concentrated Solar Power. 2012; https://doi.org/10.1115/ IMECE2012-87707.

42. Kim, H.; Kim, Y.-S.; Kwac, L.; Shin, H.; Lee, S.; Lee, U.; Shin, H. Latent Heat Storage and Thermal Efficacy of Carboxymethyl Cellulose Carbon Foams Containing Ag, Al, Carbon Nanotubes, and Graphene in a Phase Change Material. Nanomaterials $\mathbf{2 0 1 9}, 9,158$.

43. Jo, B.; Banerjee, D. Enhanced Specific Heat Capacity of Molten Salt-Based Nanomaterials: Effects of Nanoparticle Dispersion and Solvent Material. Acta Materialia $\mathbf{2 0 1 4}, 75,80-91$.

44. Shin, D.; Tiznobaik, H.; Banerjee, D. Specific Heat Mechanism of Molten Salt Nanofluids . Applied Physics Letters 2014, 104, 121914.

45. Svobodova-Sedlackova, A.; Barreneche, C.; Alonso, G.; Fernandez, A. I.; Gamallo, P. Effect of Nanoparticles in Molten Salts-MD Simulations and Experimental Study. Renewable Energy 2020, 152, 208 - 216.

46. Bolmatov, D.; Trachenko, K. Liquid Heat Capacity in the Approach from the Solid State: Anharmonic Theory. Physical Review B 2011, 84, 054106.

47. Trachenko, K.; Brazhkin, V. V. Collective Modes and Thermodynamics of the Liquid State. Reports on Progress in Physics 2015, 79, 016502. 
48. Bolmatov, D.; Brazhkin, V. V.; Trachenko, K. The Phonon Theory of Liquid Thermodynamics. Scientific Reports 2012, 2, 421.

49. Yang, C.; Dove, M. T.; Brazhkin, V. V.; Trachenko, K. Emergence and Evolution of the $k$ Gap in Spectra of Liquid and Supercritical States. Physical Review Letters 2017, 118, 215502.

50. Plimpton, S. Fast Parallel Algorithms for Short-Range Molecular Dynamics. Journal of Computational Physics 1995, 117, 1 - 19.

51. LAMMPS Molecular Dynamics Simulator. http://lammps.sandia.gov, (accessed Dec 2, 2020). 